CERN-EP/98-128

3 August 1998

\title{
LEADING-ORDER QCD ANALYSIS OF NEUTRINO-INDUCED DIMUON EVENTS
}

\section{The CHARM II Collaboration}

P. Vilain ${ }^{1)}$ and G. Wilquet ${ }^{1)}$

Inter-University Institute for High Energies (ULB-VUB), Brussels, Belgium.

R. Beyer ${ }^{2)}$, W. Flegel, T. Mouthuy ${ }^{3)}$, H. Øverås, J. Panman, A. Rozanov ${ }^{3)}$, K. Winter ${ }^{4)}$, G. Zacek ${ }^{5)}$ and V. Zacek ${ }^{6)}$

CERN, Geneva, Switzerland.

F.W. Büsser, C. Foos, L. Gerland, T. Layda, F. Niebergall, G. Rädel ${ }^{7)}$, P. Stähelin and T. Voss ${ }^{2}$

II. Institut für Experimentalphysik ${ }^{8)}$, Universität Hamburg, Hamburg, Germany.

V. Lemaître ${ }^{2)}$, D. Favart, Gh. Grégoire and E. Knoops ${ }^{9)}$

Université Catholique de Louvain, Louvain-la-Neuve, Belgium

P. Gorbunov, E. Grigoriev, V. Khovansky and A. Maslennikov ${ }^{10)}$

Institute for Theoretical and Experimental Physics, Moscow, Russia

W. Lippich, A. Nathaniel, A. Staude and J. Vogt Sektion Physik ${ }^{8)}$ der Universität München, Munich, Germany

A.G. Cocco, A. Ereditato, G. Fiorillo, F. Marchetti-Stasi, V. Palladino and P. Strolin Università "Federico II" and INFN Sezione di Napoli, Naples, Italy

A. Capone, D. De Pedis, U. Dore, A. Frenkel-Rambaldi, P.F. Loverre, D. Macina ${ }^{11)}$, G. Piredda and R. Santacesaria Università 'La Sapienza' and INFN Sezione di Roma, Rome, Italy

E. Di Capua, S. Ricciardi ${ }^{7)}$ and B. Saitta

Università di Ferrara and INFN Sezione di Ferrara, Ferrara, Italy

B. Akkus ${ }^{12)}$, E. Arik ${ }^{12)}$, M. Serin-Zeyrek, R. Sever and P. Tolun

High Energy Physics Research Centre, YEFAM, Ankara, Turkey

K. Hiller, R. Nahnhauer and H.E. Roloff ${ }^{\dagger}$

DESY-Institut für Hochenergiephysik, Zeuthen, Germany

(To be submitted to the European Physical Journal C) 


\begin{abstract}
The results of a leading-order QCD analysis of neutrino-induced charm production are presented. They are based on a sample of $4111 \nu_{\mu^{-}}$and $871 \bar{\nu}_{\mu}$-induced opposite-sign dimuon events with $E_{\mu 1}, E_{\mu 2}>6 \mathrm{GeV}, 35<E_{\nu}<290 \mathrm{GeV}$ and $Q^{2}>$ $5.5 \mathrm{GeV}^{2}$, observed in the CHARM II detector exposed to the CERN wideband neutrino and antineutrino beams. The analysis yields the value of the charm quark mass $m_{c}=1.79 \pm 0.38 \mathrm{GeV} / c^{2}$ and the Cabibbo-KobayashiMaskawa matrix element $\left|V_{c d}\right|=0.219 \pm 0.016$. The strange quark content of the nucleon is found to be suppressed with respect to non-strange sea quarks by a factor $\kappa=0.39 \pm 0.09$.
\end{abstract}

1) National Foundation for Scientific Research, Belgium.

2) Now at DESY, Hamburg, Germany.

3) Now at CPPM/IN2P3-CNRS, Marseille, France.

4) Now at Humboldt University, Berlin (Germany).

5) Now at McGill University, Montreal, Canada.

6) Now at University of Montreal, Canada.

7) Now at CERN, Geneva, Switzerland.

8) Supported by the German Bundesministerium für Forschung und Technologie (BMFT), under contract numbers $05-5 \mathrm{HH} 22 \mathrm{P}$ and $05-5 \mathrm{MU} 12 \mathrm{P}$.

9) Inter-University Institute for Nuclear Science, Belgium.

10) Now at CASPUR, Rome, Italy

11) Now at University of Geneva, Switzerland.

12) Now at Bogazici University, Istanbul, Turkey.

$\dagger$ Deceased. 


\section{$1 \quad$ Introduction}

The first neutrino-production of dimuon events was reported in 1975 by the HPWF collaboration [1]. This collaboration, and others confirming their observation $[2,3,4,5]$, explained that the origin of these events was the production of a single charm particle. In this reaction, the leading muon comes from the neutrino-nucleon charged-current interaction vertex, and the second muon comes from the semileptonic decay of the charm particle.

In twenty years of neutrino interaction studies, the understanding of these events has improved drastically. If in the 1970s such events were considered primarily as an experimental proof of the existence of the charm quark, nowadays they are used for the study of the nucleon structure and for the determination of fundamental parameters of the Standard Model. In neutrino-nucleon interactions, single charm particles can be produced both through $d c$ and $s c$ electroweak currents. The $s c$ current dominates in the antineutrino-induced dimuon events, whilst in neutrino-induced events the $d c$ and $s c$ currents have comparable contributions, as the large $d$ quark content of the nucleon compensates the Cabibbo suppression of the $d c$ transition. Therefore, using neutrino and antineutrino beams, a combined analysis of these two electroweak currents is possible, and quantities such as the suppression factor $\kappa$ of the strange quark content of the sea, the charm quark mass $m_{c}$, and the Cabibbo-Kobayashi-Maskawa (CKM) matrix element $\left|V_{c d}\right|$ can be determined.

This paper presents the results of the analysis of the dimuon events carried out by the CHARM II collaboration. The detector [6] was optimized for a dedicated study of $\nu_{\mu} e$ scattering and consists of a massive low-density target calorimeter followed by a toroidal iron muon spectrometer equipped with scintillator planes and drift chambers (Fig. 1). It was exposed to the horn-focused wide band neutrino and antineutrino beams at the CERN 450 GeV Super Proton Synchrotron (SPS).

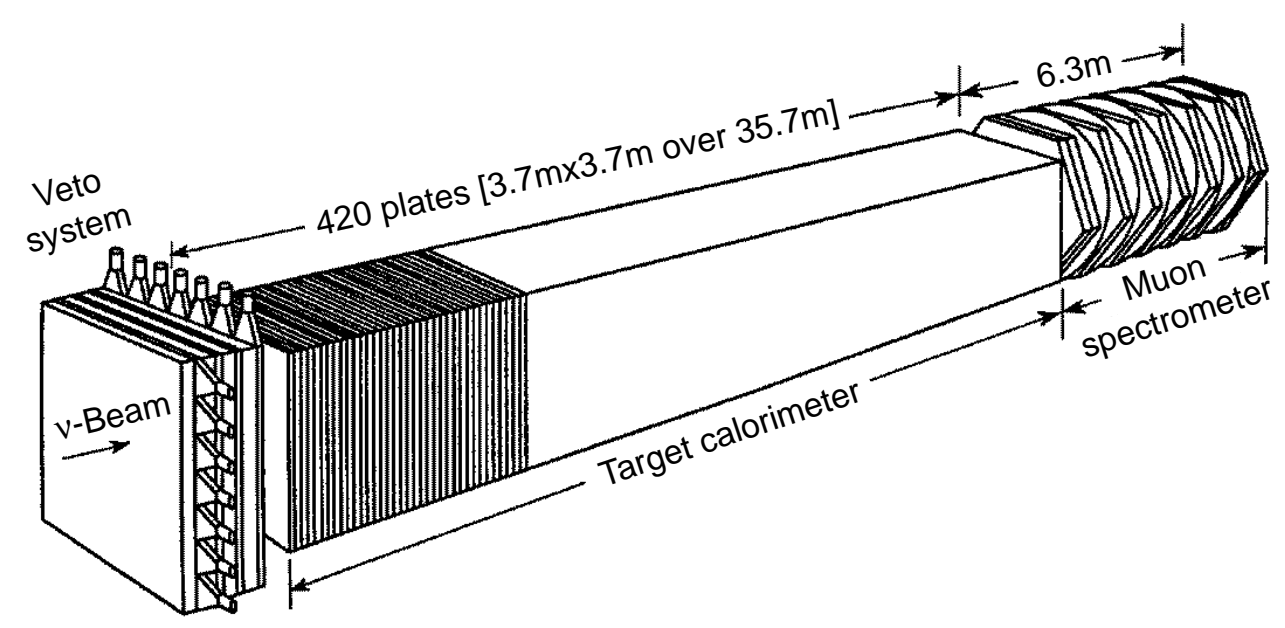

Figure 1: Schematic view of the CHARM II detector.

The target calorimeter is composed of 420 identical units with a total target mass of 692 t. Each unit contains a $4.8 \mathrm{~cm}$ glass plate followed by a plane of streamer tubes. The transverse size of a unit is $370 \times 370 \mathrm{~cm}^{2}$. The experimental signature of a dimuon event is shown in Fig. 2. Basic information which can be reconstructed includes the muon directions, the momenta and charges, and the hadronic energy. 


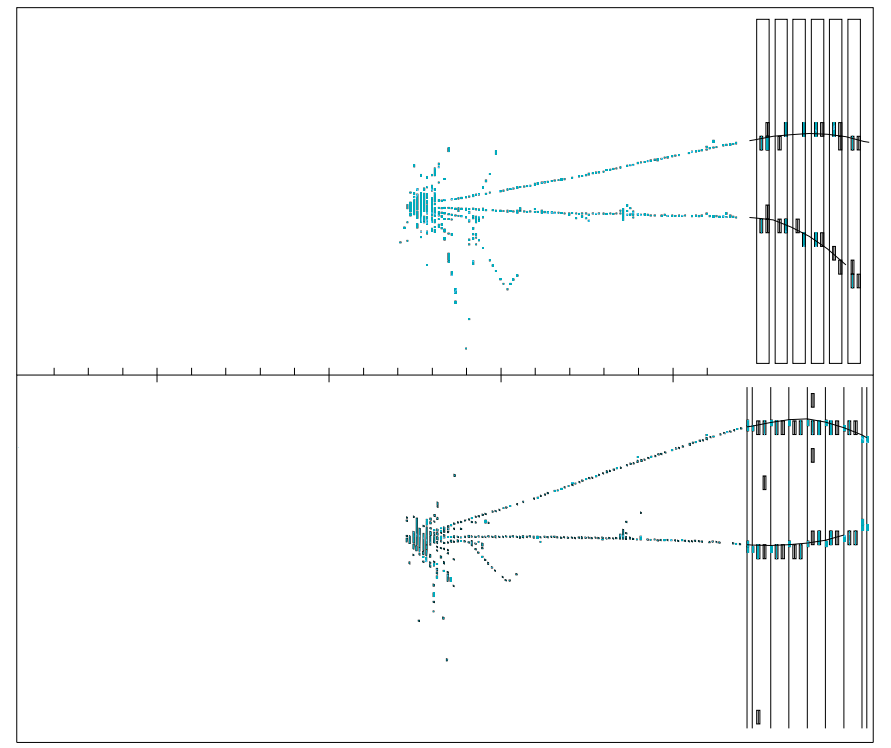

Figure 2: An example of a dimuon event in the CHARM II calorimeter. The two views refer to the horizontal and vertical projections.

In the analysis, the distributions of various kinematical variables derived from these quantities are compared with the distributions predicted by a theoretical model, taking into account the beam properties, the detector response and background contributions. The adjustment of the predicted distributions to the observed ones yields the values of the physical parameters of the model (charm quark mass $m_{c}$, strange quark content of a nucleon, CKM matrix element $\left.\left|V_{c d}\right|\right)$, together with the tuning parameters of the phenomenological part of the model.

This paper is organized as follows: in Section 2, all the theoretical prerequisites are introduced; Section 3 gives details about the neutrino flux normalization; Section 4 describes the data selection based on kinematical variables specific to dimuon production and details about detector simulation; Section 5 is devoted to background determination and Section 6 summarizes the results of the fit. Section 7 contains a discussion, and a comparison with the results of other experiments. New estimates of $\left|V_{c d}\right|$ and of the electroweak mixing parameter $\sin ^{2} \theta_{W}$ are presented. The last section summarizes the results.

\section{Theoretical framework}

\subsection{The leading-order charm production cross-section}

For this analysis, we have chosen the leading order (LO) calculation of the deep inelastic neutrino scattering (DIS) cross-section by M.A.G. Aivazis et al. [7]. These authors use the helicity formalism to take into account, in a natural way, various scales related to this reaction (in particular, nucleon and quark masses). In this approach, the LO cross-section for the reaction $\nu_{\mu} N \rightarrow \mu^{-} c X$ and $\bar{\nu}_{\mu} N \rightarrow \mu^{+} \bar{c} X$ takes the form: 


$$
\begin{aligned}
\frac{d^{2} \sigma_{c}^{\nu(\bar{\nu})}}{d x d y}= & \frac{G_{F}^{2} y Q^{2}}{\pi\left(1+Q^{2} / M_{W}^{2}\right)^{2}}\left(\left|V_{c d}\right|^{2} \stackrel{(-)}{d}(\chi)+\left|V_{c s}\right|^{2} \stackrel{(-)}{s}(\chi)\right) \times \\
& {\left[\left(\frac{1+\cosh \psi}{2}\right)^{2}+\frac{m_{c}^{2}}{2 Q^{2}} \frac{\sinh ^{2} \psi}{2}\right]\left[1+\delta_{r}\left(E_{\nu}, \chi, y\right)\right], }
\end{aligned}
$$

where $G_{F}$ is the Fermi coupling constant, $\left|V_{c d}\right|$ and $\left|V_{c s}\right|$ are the CKM mixing matrix elements, $x$ and $y$ are the usual Bjorken variables, $d$ and $s$ are the momentum distributions of the corresponding quarks in a nucleon, $Q^{2}$ is the square of the 4-momentum transfer, and $E_{\nu}$ is the neutrino energy. $\delta_{r}\left(E_{\nu}, \chi, y\right)$ is the QED radiative correction as calculated by Bardin [8]. We assume the PDG value [9] of $\left|V_{c d}\right|=0.221 \pm 0.003$ and $\left|V_{c s}\right|=0.9743 \pm$ 0.0008 .

The scaling variable $\chi(0<\chi<1)$ is defined by

$$
\chi=\eta \frac{\left(Q^{2}-m_{q}^{2}+m_{c}^{2}\right)+\Delta\left(-Q^{2}, m_{q}^{2}, m_{c}^{2}\right)}{2 Q^{2}},
$$

where $m_{q}$ is the $s$ or the $d$ quark mass, $\eta$ is

$$
\eta=\left[\frac{1}{2 x}+\sqrt{\frac{1}{4 x^{2}}+\frac{M^{2}}{Q^{2}}}\right]^{-1}
$$

and $\Delta$ is the kinematical function

$$
\Delta(a, b, c)=\sqrt{a^{2}+b^{2}+c^{2}-2(a b+b c+c a)} .
$$

$M$ is the nucleon mass. For $Q^{2} \gg M^{2}, \chi$ becomes identical to $x$. The hyperbolic angle $\psi$ is defined as

$$
\cosh \psi=\frac{E_{\nu}+E_{\mu}}{\sqrt{Q^{2}+\nu^{2}}}
$$

where $E_{\nu}$ is the neutrino energy, $E_{\mu}$ is the leading muon energy, and $\nu=E_{\nu}-E_{\mu}$. As the target calorimeter of CHARM II is made of an isoscalar material, the $d$-quark distribution function is equal to

$$
d=\frac{d^{p}+u^{p}}{2}
$$

with $u^{p}$ and $d^{p}$ being the up and down quark momentum distribution functions in the proton.

In (1), the quark distribution functions evolve in $Q^{2}$ according to the GribovLipatov-Altarelli-Parisi equation [10, 11].

\section{$2.2 \quad$ Parton distributions}

In this analysis, the free parameters are the same as in the analysis of the CCFR collaboration [12] and the fitting procedure is similar. However, unlike CCFR, who extracted the quark distribution functions from their own measurement of $F_{2}$ and $x F_{3}$, we use the valence and total sea quark distribution functions obtained from the global fit to DIS data by the CTEQ group [13]. One of the outputs of the present analysis is the strange content of the nucleon, $\kappa$, defined as follows:

$$
\kappa=\frac{\int_{0}^{1}\left[x s\left(x, \mu_{0}^{2}\right)+x \bar{s}\left(x, \mu_{0}^{2}\right)\right] d x}{\int_{0}^{1}\left[x \bar{u}\left(x, \mu_{0}^{2}\right)+x \bar{d}\left(x, \mu_{0}^{2}\right)\right] d x},
$$


where $\mu_{0}$ is an arbitrary reference scale (we choose $\mu_{0}^{2}=20 \mathrm{GeV}^{2}$ ) ${ }^{1}$ ). We require the total sea distribution $x \bar{q} \equiv x \bar{u}+x \bar{d}+x \bar{s}$ to reproduce $x \bar{q}_{0} \equiv x \bar{u}_{0}+x \bar{d}_{0}+x \bar{s}_{0}$ from CTEQ [13], but allow $x \bar{s}$ (and hence, $x \bar{u}+x \bar{d}$ ) to deviate from the CTEQ parametrization by introducing an extra $x$-dependence through a free parameter $\alpha$ :

$$
\begin{gathered}
x \bar{q}\left(x, \mu^{2}\right)=x \bar{q}_{0}\left(x, \mu^{2}\right) \\
x \bar{s}\left(x, \mu^{2}\right)=x s\left(x, \mu^{2}\right)=A_{s}(1-x)^{\alpha} \frac{x \bar{u}\left(x, \mu^{2}\right)+x \bar{d}\left(x, \mu^{2}\right)}{2} \\
\frac{x \bar{u}\left(x, \mu^{2}\right)+x \bar{d}\left(x, \mu^{2}\right)}{2}=\frac{x \bar{q}_{0}\left(x, \mu^{2}\right)}{2+A_{s}(1-x)^{\alpha}} .
\end{gathered}
$$

The strange sea normalization $A_{s}$ is fixed by (2) and (3) and, for any values of the free parameters $\kappa$ and $\alpha$, can be determined from the equation

$$
A_{s}=\kappa \frac{\int_{0}^{1} \frac{x \bar{q}_{0}}{2+A_{s}(1-x)^{\alpha}} d x}{\int_{0}^{1} \frac{(1-x)^{\alpha} x \bar{q}_{0}}{2+A_{s}(1-x)^{\alpha}} d x} .
$$

Note that $A_{s}$ becomes identical to $\kappa$ if $\alpha=0$.

\subsection{Fragmentation and decays}

A phenomenological approach is used for the description of the fragmentation process: the fraction $z$ of the $c$-quark longitudinal momentum taken over by the charm hadron is assumed to be distributed according to the Peterson fragmentation function [14]:

$$
D_{h / c}(z, \epsilon) \propto z^{-1}\left(1-\frac{1}{z}-\frac{\epsilon}{(1-z)}\right)^{-2},
$$

where $\epsilon$ is considered as a free parameter which will be fitted. The same fragmentation function is used for all charm hadrons.

For the transverse momentum of charm hadrons with respect to the $W$-boson direction an exponential distribution is assumed:

$$
\frac{d N}{d p_{t}^{2}} \propto e^{-b p_{t}^{2}}
$$

with $b=1.7 \pm 1.5$. The central value was determined by averaging the parameter values used by the E531 [15], CDHS [16] and CCFR [12] collaborations, and the error covers the entire range of these values.

To model the charm hadron species, we use the production fractions (Table 1) obtained from a re-analysis of the E531 data by T. Bolton [17]. The E531 data covers the neutrino energy range of $30 \mathrm{GeV}$ to $200 \mathrm{GeV}$, similar to that of the present analysis.

For charm hadron decay simulation, we use exclusive semileptonic modes summarized in Table 2 , in which $D$ stands for $D^{ \pm}, \bar{D}^{0}$ and $D_{s}^{ \pm}$, and $\pi \mu \nu$ stands for all Cabibbosuppressed modes. The corresponding relative branching ratios are derived from the 1994 PDG [18]. Decay matrix elements are not taken into account in decay modelling.

1) The dependence of $\kappa$ on the scale $\mu_{0}$ is expected to be small within the limited $\left(x, Q^{2}\right)$-domain of this analysis, and therefore the fact that we used $\mu_{0}^{2}=20 \mathrm{GeV}^{2}$ to define $\kappa$ will no longer be mentioned. 
Table 1: Production fractions of the charm hadron species used in the model.

\begin{tabular}{|c|c|}
\hline Particle & Production fraction \\
\hline$D^{0}$ & $0.60 \pm 0.06$ \\
$D^{+}$ & $0.26 \pm 0.06$ \\
$D_{s}^{+}$ & $0.07 \pm 0.05$ \\
$\Lambda_{c}^{+}$ & $0.07 \pm 0.04$ \\
\hline
\end{tabular}

Table 2: The exclusive semileptonic decay modes of charm hadrons used in the model.

\begin{tabular}{|c|c|}
\hline Mode & Relative branching ratio \\
\hline$D \rightarrow \bar{K} \mu \nu$ & $0.60 \pm 0.03$ \\
$D \rightarrow \bar{K}^{*} \mu \nu$ & $0.33 \pm 0.03$ \\
$D \rightarrow \pi \mu \nu$ & $0.07 \pm 0.02$ \\
\hline$\Lambda_{c} \rightarrow p \mu X$ & $0.56 \pm 0.28$ \\
$\Lambda_{c} \rightarrow \Lambda \mu X$ & $0.44 \pm 0.16$ \\
\hline
\end{tabular}

The average semi-muonic decay branching ratio of charm hadrons is evaluated in Ref. [17] as

$$
\bar{B}_{\mu}=0.0919 \pm 0.0094
$$

where the error is determined by the uncertainties in the charm hadron species fractions (0.0085) and semi-leptonic branching ratios (0.0041), added here in quadrature. In the present analysis we consider $B_{\mu}$ as a free parameter which will be fitted, and use the measured value of $\bar{B}_{\mu}$ only for the independent determination of $\left|V_{c d}\right|$ (see Section 6.5).

Finally, the dimuon cross-section has the form:

$$
\frac{d^{3} \sigma^{\nu(\bar{\nu}) \rightarrow 2 \mu}}{d x d y d z}=\frac{d^{2} \sigma_{c}^{\nu(\bar{\nu})}}{d x d y}\left(\kappa, \alpha, m_{c}, E, x, y\right) D(\epsilon, z) B_{\mu} .
$$

The quantities $\kappa, \alpha, m_{c}, \epsilon$ and $B_{\mu}$, introduced in this and previous sub-sections, constitute the set of main model parameters which will be determined from the data.

\section{$3 \quad$ Normalization}

The absolute cross-section normalization requires the knowledge of the total neutrino flux, energy spectra and compositions for both neutrino and antineutrino beams.

\subsection{Neutrino spectra}

The $\nu_{\mu}$ and $\bar{\nu}_{\mu}$ spectra are obtained by unfolding the resolution function from the muon spectra observed in the quasi-elastic reactions:

$$
\begin{gathered}
\nu_{\mu}+n \rightarrow \mu^{-} p \\
\overline{\nu_{\mu}}+p \rightarrow \mu^{+} n .
\end{gathered}
$$

These processes are particularly suitable for this purpose because, firstly, at high energies their cross-sections are almost energy independent and, secondly, the true muon 
momentum is very close to the neutrino energy. Apart from reactions (7), events with a single muon and no (or little) visible hadronic activity may come from background processes like $\Delta$ and $N^{*}$ resonance production and (for neutrinos) inverse muon decay. These background contributions are taken into account in the unfolding procedure.

The result of the unfolding is shown in Fig. 3, together with computed $\nu_{e}$ and $\left(\bar{\nu}_{e}\right)$ spectra. Table 3 gives the mean energies and fractions of the main contaminating neutrino species for both beams.
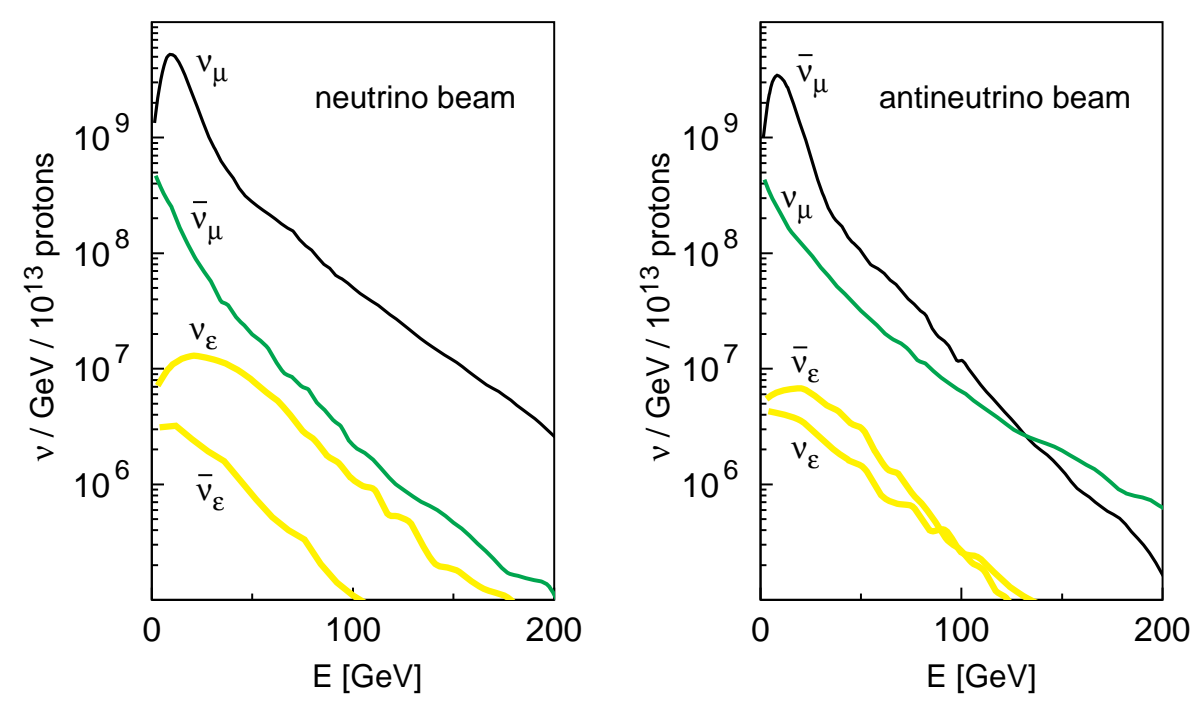

Figure 3: Energy spectra of the neutrino and antineutrino beams. The $\nu_{\mu}$ and $\bar{\nu}_{\mu}$ spectra come from the unfolding of the measured quasi-elastic events energy distributions, and $\nu_{e}$ and $\bar{\nu}_{e}$ - from the beam simulation.

Table 3: Experimentally determined neutrino beam properties.

\begin{tabular}{|c||c|c|c|}
\hline & $\left\langle E_{\nu_{\mu}}\right\rangle, \mathrm{GeV}$ & $\left\langle E_{\bar{\nu}_{\mu}}\right\rangle, \mathrm{GeV}$ & Contamination \\
\hline$\nu$ & $23.7 \pm 0.3$ & $19.2 \pm 0.5$ & $\bar{\nu}_{\mu}:(7.2 \pm 0.4) \%$ \\
\hline $\bar{\nu}$ & $26.3 \pm 0.6$ & $19.1 \pm 0.2$ & $\nu_{\mu}:(13.6 \pm 0.7) \%$ \\
\hline
\end{tabular}

\subsection{Neutrino and antineutrino fluxes}

The neutrino flux is determined on the basis of the deep-inelastic charged-current (CC) reaction:

$$
\nu_{\mu}+N \rightarrow \mu^{-}+X
$$

The cross-section of this reaction is known with good accuracy [19]:

$$
\hat{\sigma}_{\text {incl }}^{\mathrm{CC}}(\nu N)=\sigma_{\text {incl }}^{\mathrm{CC}}(\nu N) / E_{\nu}=(0.677 \pm 0.014) \times 10^{-38} \mathrm{~cm}^{2} / \mathrm{GeV}
$$

and therefore, provided the beam energy spectrum is known, the total neutrino flux through a given fiducial volume can be derived from the total number of neutrino-induced $\mathrm{CC}$ events in this volume. In this experiment, the number of $\mathrm{CC}$ events is deduced from the observed number $N_{\text {incl }}^{\mathrm{MB}}$ of 'minimum bias' triggers requiring a shower energy above 
a threshold of about $3 \mathrm{GeV}$. Taking into account the neutrino beam composition and contributions from charged-current and neutral-current (NC) interactions, we have:

$$
N_{\text {incl }}^{\mathrm{CC}}=\frac{N_{\text {incl }}^{\mathrm{MB}} \hat{\sigma}_{\text {incl }}^{\mathrm{CC}}\left(\nu_{\mu} N\right)\left\langle E_{\nu_{\mu}}\right\rangle}{\sum_{p} \sum_{\nu_{i}} \int \frac{d \phi}{d E_{\nu_{i}}} A_{\nu_{i}}^{p}\left(E_{\nu_{i}}\right) \hat{\sigma}_{\text {incl }}^{p}\left(\nu_{i} N\right) E_{\nu_{i}} d E_{\nu_{i}}},
$$

where $\nu_{i}$ represents the neutrino beam components $\left(\nu_{\mu}, \bar{\nu}_{\mu}, \nu_{e}\right.$ and $\left.\bar{\nu}_{e}\right), p$ denotes the processes involved (CC and $\mathrm{NC}$ ), and $d \phi / d E_{\nu_{i}}$ are neutrino beam spectra. The acceptance functions $A\left(E_{\nu_{i}}\right)$ are determined by a detailed Monte Carlo simulation of $\nu$-induced hadronic showers.

The estimated accuracy of this method is $4 \%$. The resulting number of chargedcurrent events,

$$
N_{\text {incl }}^{\mathrm{CC}}=(4.57 \pm 0.18) \times 10^{7},
$$

corresponds to the average total $\nu_{\mu}$ flux $\Phi_{\nu_{\mu}}=(9.1 \pm 0.4) \times 10^{11} \mathrm{~cm}^{-2}$ through a fiducial mass of about 520 t.

The use of antineutrino dimuon-induced events is of great importance for extracting the strange quark content of the nucleon. The total flux of muon antineutrinos in the $\bar{\nu}$ beam, $\Phi_{\bar{\nu}_{\mu}}$, has been measured relative to $\Phi_{\nu_{\mu}}$ using five different methods.

In the first method, the minimum bias analysis has been extended to the antineutrino beam providing a determination of $N_{\text {incl }}^{\mathrm{CC}}\left(\bar{\nu}_{\mu}\right) / N_{\text {incl }}^{\mathrm{CC}}\left(\nu_{\mu}\right)$ and, hence, the relative $\bar{\nu}_{\mu}$ flux.

The second method makes use of fully reconstructed CC events selected in a restricted fiducial volume and in the kinematical domain where the Monte Carlo simulation reproduces data well and the experimental uncertainties are minimal. The relative flux is determined by a simultaneous fit of the simulated distributions in the kinematical variables $E_{\text {had }}, E_{\mu}, E_{\text {tot }}$ and $y$ to the ones measured in the $\nu_{\mu}$ and $\bar{\nu}_{\mu}$ beams. Figure 4 illustrates the results of the fit. The only systematic uncertainties in common with the previous method are related to the ratio of the total $\nu_{\mu}$ and $\bar{\nu}_{\mu}$ cross-sections and the mean neutrino beam energy. However, these errors are relatively small compared to other errors specific to both methods, so the methods are virtually independent.

The third method uses the quasi-elastic sample. The analysis makes use of the fact that the cross-sections of neutrino- and antineutrino-induced quasi-elastic reactions are equal at $Q^{2}=0$. Provided the selection efficiencies are similar for neutrino and antineutrino beams, the flux ratio can be deduced from the ratio of event counts at $Q^{2}=0$. This ratio can only be calculated by an extrapolation because of the presence of the inverse muon decay and Pauli suppression at low $Q^{2}$. Figure 5 shows the $Q^{2}$ distributions for Monte Carlo events and for data in the neutrino and antineutrino beams. The mixture of quasi-elastic scattering and resonance production has been fixed in the Monte Carlo model according to their cross-sections. The number of simulated events are normalized to the data in the region $0.05-0.2 \mathrm{GeV}^{2}$.

The fourth method uses the coherent neutral pion sample. Data selection is similar to the $\nu_{\mu} e^{-}$scattering sample which is described elsewhere [20, 21]. With the crosssections for coherent $\pi^{0}$ production being equal for neutrino and antineutrino at $Q^{2}=0$, in accordance with the Adler theorem [22], a procedure similar to that for quasi-elastic reactions has been used to extract the relative neutrino flux.

Finally, the fifth method uses measurements of the muon flux in the iron shielding downstream of the decay region of the neutrino beam line. The relation between the 

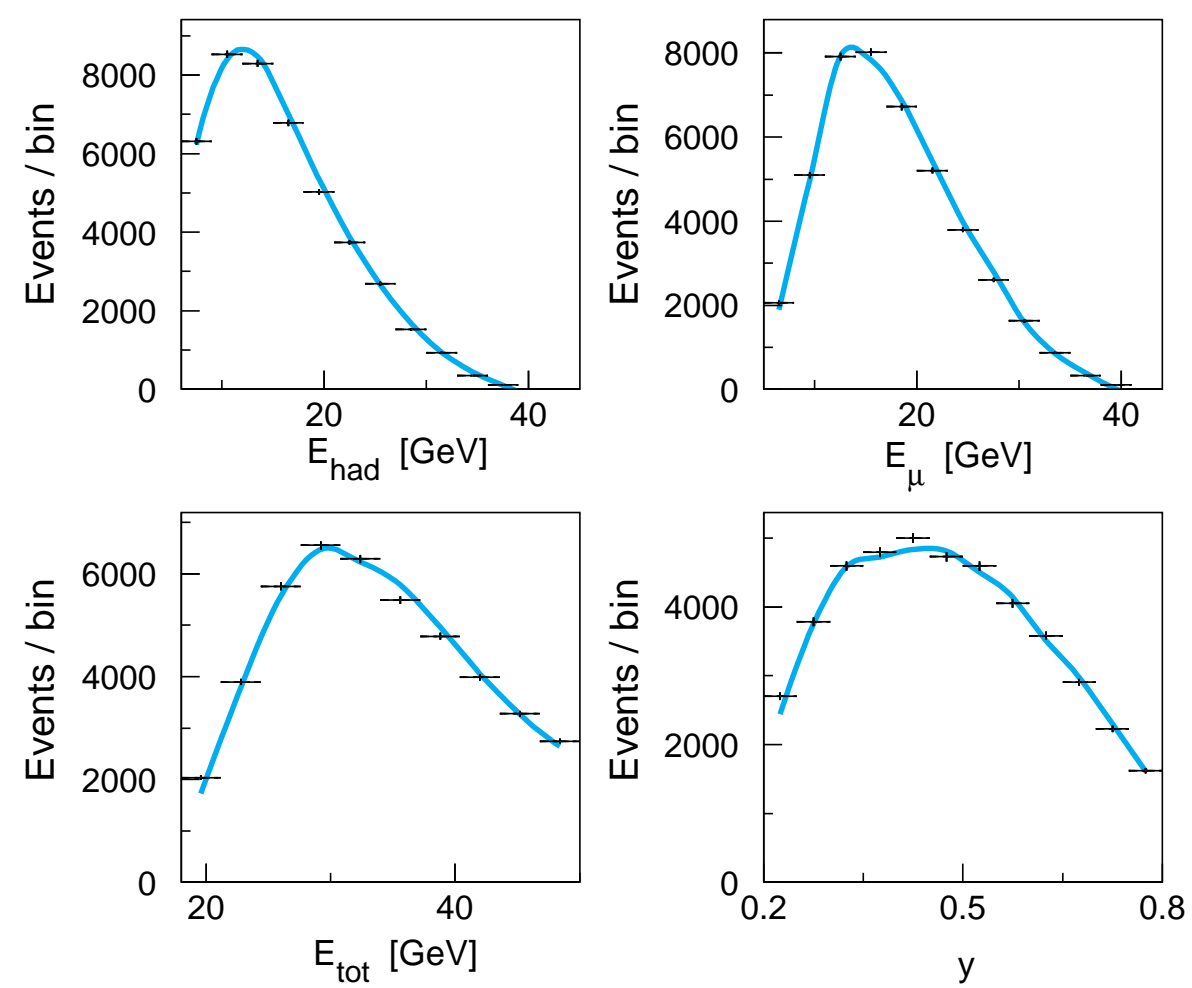

Figure 4: The fit of simulated distributions (lines) to the data (crosses), for $\nu_{\mu}$-induced CC events.
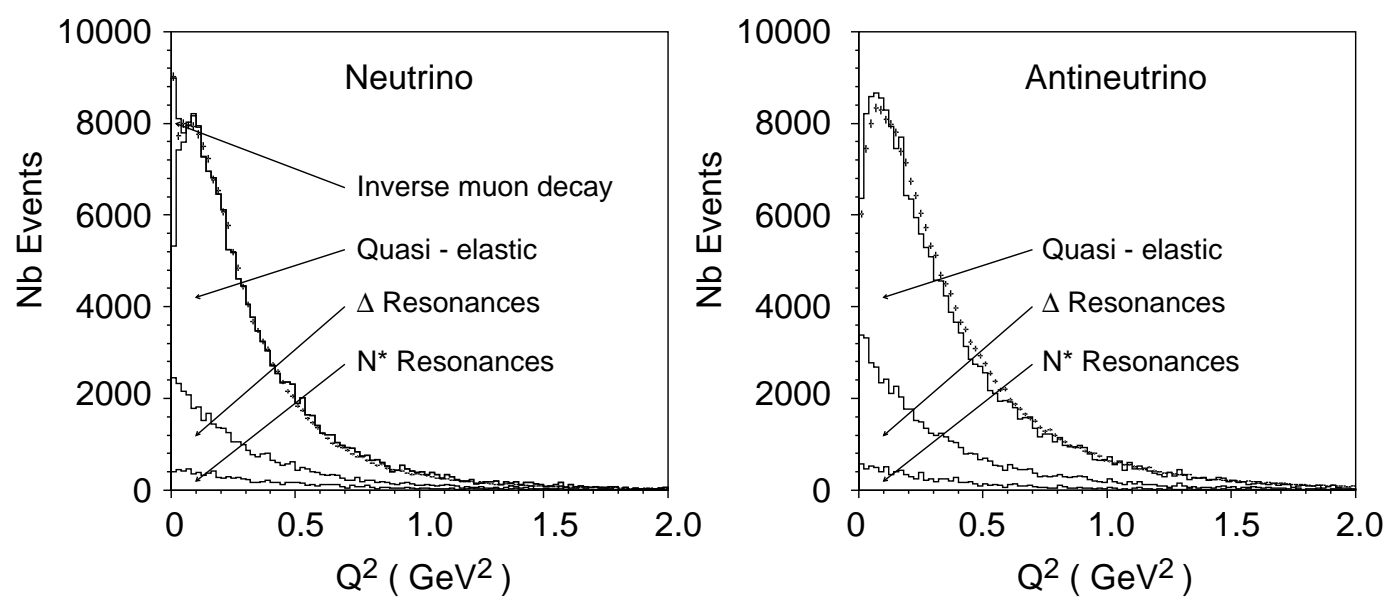

Figure 5: Simulated $Q^{2}$ distributions of events from quasi-elastic scattering and background reactions (with different contributions shown stacked on top of each other), compared with experimental data (crosses), for neutrino and antineutrino beams. 
measured muon flux and the corresponding neutrino flux is established by a Monte Carlo simulation of the neutrino beam.

The results for the relative neutrino flux $f$ coming from these five independent normalization methods are statistically compatible. The mean value is:

$$
f=\frac{\Phi_{\bar{\nu}_{\mu}}}{\Phi_{\nu_{\mu}}}=1.270 \pm 0.027
$$

\section{Data selection and detector response simulation}

\subsection{Data recording and dimuon trigger}

The detector was exposed to the neutrino beam between 1987-1991. Sharing between the neutrino and antineutrino data-taking period was optimized in order to have approximately equal numbers of $\nu_{\mu} e$ - and $\bar{\nu}_{\mu} e$-scattering events, the main reaction studied by CHARM II [21]. The beam polarity was changed every two or three days in order to reduce possible systematic effects caused by beam instabilities.

During every $6 \mathrm{~ms}$ long neutrino spill $\sim 500$ neutrino interactions, $\sim 300$ beam related muons and $\sim 300$ cosmic muons occurred in the detector. The dimuon trigger $[6]$ required a clear two-track topology in at least one of the two calorimeter projections, with a minimum distance between the tracks of $\sim 10 \mathrm{~cm}$ over at least 30 planes.

\subsection{Data reduction and reconstruction}

Dimuon events were accepted for the final analysis if they satisfied the following selection criteria:

(1) the vertex, located by extrapolating the muon tracks through the hadronic shower, had to be between calorimeter planes 25 and 390 (with the total target extending from plane 21 to 440) to ensure a full longitudinal containment of the hadronic shower and to fulfil the longitudinal requirement of the dimuon trigger;

(2) laterally, the vertex had to be within a square of $320 \times 320 \mathrm{~cm}^{2}$, to ensure lateral containment of the hadronic shower;

(3) both muons had to be well reconstructed, with the closest approach between them being less than $5 \mathrm{~cm}$ (to reject overlays of two CC events as well as obvious muons from the decay of shower hadrons).

For each event, the reconstructed muon parameters at the vertex $\left(\vec{p}_{i}, E_{i}, \vec{r}_{i}=\vec{p}_{i} /\left|p_{i}\right|\right.$, $i=1,2)$ and the shower energy, $E_{\text {had }}$, were used to derive the following kinematical variables:

$-E_{\nu v}=E_{1}+E_{2}+E_{\text {had }}$, the visible neutrino energy;

- $P t_{w 1 v}$ and $P t_{w 2 v}$, the transverse muon momenta with respect to the reconstructed $W$-boson direction. The latter is defined by the vector $E_{\nu v} \vec{x}-E_{1} \vec{r}_{1}$, where $\vec{x}$ and $\vec{r}$ are unitary vectors parallel to the beam and muon directions, and the index 1 denotes the assumed leading muon (see below);

- $Q_{v}^{2}=2 E_{\nu v}\left(E_{1}-\vec{p}_{1} \cdot \vec{x}\right)-m_{\mu}^{2}$, where $\vec{p}_{1}$ is the leading muon 3-momentum;

- $\nu_{v}=E_{2}+E_{\text {had }}$, the visible $W$-boson energy;

- $W_{v}^{2}=M^{2}+2 M \nu_{v}-Q_{v}^{2}$, the square of the visible hadronic invariant mass ( $M$ is the nucleon mass); 
$-m_{12}=\sqrt{\left(E_{1}+E_{2}\right)^{2}-\left(\vec{p}_{1}+\vec{p}_{2}\right)^{2}}$, the invariant mass of the muons;

- $x_{v}=Q_{v}^{2} / 2 M \nu_{v}$, the visible Bjorken $x$ variable;

- $y_{v}=\nu_{v} / E_{\nu v}$, the visible Bjorken $y$ variable;

$-z_{l v}=E_{2} /\left(E_{2}+E_{\text {had }}\right)$

- $P t_{1}$ et $P t_{2}$, transverse muon momenta with respect to the neutrino beam direction;

- $\phi_{12}$, the angle between the transverse muon momenta.

Because of the admixture of 'wrong-helicity' neutrinos $\left(\bar{\nu}_{\mu}\right.$ 's in the neutrino beam and $\nu_{\mu}$ 's in the antineutrino beam) the leading muon produced by a neutrino had to be identified in each event by kinematical criteria, rather than by the sign of charge. We defined the leading muon as the one having the highest transverse momentum with respect to the $W$-boson direction, reconstructed accordingly. Events with a leading muon candidate of the wrong sign were rejected ${ }^{2)}$.

\subsection{Kinematical selection}

The final sample is obtained by imposing the kinematical selection described below:

- $E_{1}, E_{2}>6 \mathrm{GeV}$ and $E_{\text {had }}>5 \mathrm{GeV}$

These criteria were needed to ensure good quality reconstruction and good control over efficiencies. The high muon energy criterion also drastically reduces the meson decay background.

$-x_{v}<0.5, Q_{v}^{2}>5.5 \mathrm{GeV}^{2}$ and $E_{\nu v}>35 \mathrm{GeV}$

Criteria on $x_{v}$ and $Q^{2}$ were dictated by the choice of the parton distribution parametrization, whilst the cut on $E_{\nu v}$ was to ensure a consistent description of the fragmentation process by the E531 and $e^{+} e^{-}$data. The average $Q_{v}^{2}$ after this cut is $\left\langle Q_{v}^{2}\right\rangle_{\nu}=17.9 \mathrm{GeV}^{2}$ and $\left\langle Q_{v}^{2}\right\rangle_{\bar{\nu}}=12.7 \mathrm{GeV}^{2}$.

- for antineutrinos only: $z_{l v}<0.7$ and $P t_{w 2 v}<1.3 \mathrm{GeV} / c$. These two criteria provide a final rejection of the $\nu_{\mu}$-induced background events remaining after the leading muon selection procedure.

A total of 4111 neutrino- and 871 antineutrino-induced events survived this selection.

\subsection{Monte Carlo simulation of the experiment}

A full-scale simulation of neutrino events in the CHARM II detector was performed using the LEPTO-LUND [23] package (for primary interactions) and the GEANT [24] package (for the detector geometry description, tracking and simulation of the detector response). A comparison of the simulated detector response with data is described in Ref. [25].

However, the full-scale simulation was too slow to be used in the iterative fitting procedure described in Section 6 and therefore, fast detector response simulation functions were used in the present analysis. These functions were adjusted to obtain distributions of the 'visible' kinematical variables comparable with those from the full GEANT simulation of the detector, as shown in Fig. 6. This figure also illustrates the three main sources of event losses. The geometrical acceptance is responsible for the losses far away from the

${ }^{2)}$ In the case of same-sign dimuons used for the background measurement (Section 5), the $W$-direction was calculated assuming the most energetic muon to be leading, and no further rejection was carried out. 
muon spectrometer. The losses near the spectrometer are due to the trigger condition requiring two distinct tracks in at least 30 calorimeter planes. Finally, the spectrometer hardware and reconstruction software inefficiencies are responsible for the fall-off of the spectrum at low muon energies.
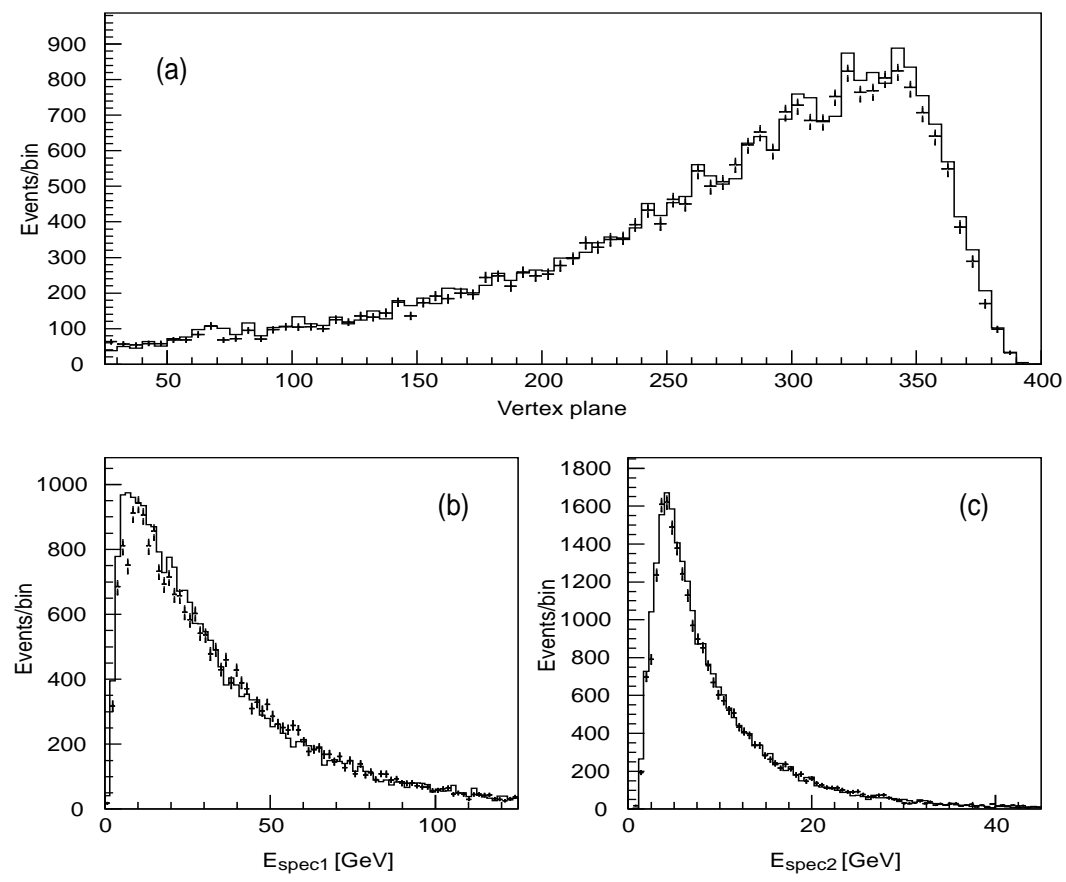

Figure 6: A comparison of the fast and full detector simulations applied to the same input sample of generated dimuon events: (a) event vertex plane distribution; (b) leading, and (c) decay muon energy distributions.

\section{$5 \quad$ Background}

\subsection{Sources}

The main background to opposite-sign dimuon events from charm production is caused by muonic decays of pions and kaons produced in CC events, either directly (vertex hadrons) or during the shower development (shower hadrons).

The background level depends on the probability of a pion or a kaon decaying before it interacts in the calorimeter. Considering only vertex hadrons, the number of background events per neutrino-nucleon CC interaction is proportional to

- the mean hadron multiplicity $\left\langle N_{h}\right\rangle$ per CC event, with the hadron energy above a threshold of $\simeq 5 \mathrm{GeV}$ (correlated with the muon energy cut used for the dimuon event selection);

- the inverse of the meson decay length $\lambda_{\text {dec }}$;

- the average nuclear interaction length $\lambda_{\text {int }}$, which in the case of pions was determined experimentally (with a $\pi^{-}$test beam) as $75 \pm 6 \mathrm{~cm}$.

Using the values in Table 4, we can roughly estimate the background rate for vertex mesons near the threshold of $5 \mathrm{GeV}$. Although for more energetic mesons the prompt decay 
background drops rapidly, due to both decreasing mean multiplicity and growing $\lambda_{\text {dec }}$, the probability of producing secondary (shower) hadrons with an energy above threshold increases, rendering this background source important for the entire energy spectrum of mesons. Sections 5.2 and 5.3 explain the background determination procedure in more detail.

Table 4: A rough estimate of the prompt meson decay background at $5 \mathrm{GeV}$

\begin{tabular}{|c|c|c|c|c|c|c|}
\hline$h$ & $\gamma$ & $\left\langle N_{h}\right\rangle$ & $\lambda_{\text {int }}$ & $c \tau$ & Muonic decay branching & Background/CC \\
\hline \hline$\pi$ & 36 & $\sim 1$ & 80 & 780 & $\sim 1$ & $\sim 3 \times 10^{-3}$ \\
\hline$K$ & 10 & $\sim 0.3$ & 90 & 371 & $\sim 0.65$ & $\sim 4 \times 10^{-3}$ \\
\hline
\end{tabular}

The contribution of other sources, such as the production of resonances or neutral strange particles, the pair production of charm particles, the diffractive production of strange charm mesons and coherent muon pair production, was found to be negligible because of the small cross-sections or the imposed kinematical selection.

\subsection{Evaluation of the background induced by pion and kaon decays}

An important property of the $\pi, K$-decay background is that it contributes in a similar way to same- and opposite-sign dimuons. On the other hand, same-sign dimuon production is dominated by this background. Therefore, same-sign dimuon samples $\left(\mu^{-} \mu^{-}\right.$ in the $\nu$ runs, $\mu^{+} \mu^{+}$in the $\bar{\nu}$ runs) were used to tune the parameters of the background simulation model which are insensitive to the meson charge. This was carried out as follows:

- with the LEPTO-LUND generator, neutrino interactions were generated according to known beam spectra;

- secondary interactions of vertex and shower hadrons were simulated according to the experimental data on inclusive pion production in $\pi N$ interactions [26];

- for each charged pion or kaon, the decay probability was calculated and, when a decay occurred, the produced muon was generated;

- the model parameters, including the non-strange fragmentation description and the normalization factors described below, were tuned to reproduce the distributions of the kinematical quantities observed for same-sign dimuons.

The secondary hadrons were generated using a fragmentation function of the form:

$$
D_{h} \propto z^{0.05} e^{-a z}
$$

and assuming the transverse momentum distribution to be

$$
d N_{h} / d P_{t} \propto e^{-c P_{t}^{2}} .
$$

Parameters $a$ and $c$ were adjusted to reproduce the observed distributions of $z_{l v}$ and $P_{t w 2 v}$ of the same-sign dimuons. The favoured ${ }^{3}$ ) and unfavoured meson relative multiplicity is assumed to be the same as for the production vertex. This assumption is justified

3) A favoured (unfavoured) meson is defined as the one having the same (opposite) electric charge as the incoming particle. 
by the similarity of the fragmentation properties of $W^{ \pm} N \rightarrow h^{ \pm} X$ and $\pi^{ \pm} N \rightarrow \pi^{ \pm} X$ processes [27].

In addition, four normalization factors: the relative vertex pion and kaon contributions $\left(b_{\pi}\right.$ and $\left.b_{k}\right)$, the shower hadron contribution $\left(b_{\mathrm{sh}}\right)$, and the contribution of the antineutrino sample relative to the neutrino sample $\left(b_{\bar{\nu} / \nu}\right)$ were fitted in order to obtain the best possible match between the observed $E_{\nu v}$, the $x_{v}$ and the $z_{l v}$ distributions.

The resulting values of these parameters are given in Table 5. A unity value for normalization $b$-factors would indicate that LEPTO-LUND provided correct weighting with its default internal settings. Given a typical $20 \%$ uncertainty $^{4}$, the only parameter which is significantly off unity is $b_{K}$, an indication of a 'deficit' of kaons among Monte Carlo simulated vertex hadrons ${ }^{5)}$.

Table 5: Adjustment parameters of the background simulation model

\begin{tabular}{|c|c|c|c|c|c|}
\hline$a$ & $c$ & $b_{\pi}$ & $b_{K}$ & $b_{\mathrm{sh}}$ & $b_{\bar{\nu} / \nu}$ \\
\hline 3 & 1.4 & 1.2 & 1.6 & 0.9 & 1.2 \\
\hline
\end{tabular}

The result of the same-sign dimuon simulation is illustrated in Figs. 7 and 8, showing a decent agreement between same-sign data and simulation after Monte Carlo program tuning.

As a further check, the multiplicity of the LEPTO-LUND generated showers was compared with data from BEBC [28] obtained with a similar beam and a $\mathrm{H}_{2}-\mathrm{Ne}$ filling. The $\mathrm{H}_{2}-\mathrm{Ne}$ filling has a mean atomic weight close to the one of the CHARM II target. Figure 9 shows the comparison between the LEPTO-LUND and the BEBC pion and hadron multiplicities in the forward hemisphere. Because of the muon energy cut applied in this analysis, these forward mesons contribute to most of the expected background.

The assumed meson interaction lengths are shown in Fig. 10 as a function of the meson momentum. These curves are derived from the meson-nucleon cross-sections quoted in Ref. [9] and normalized to reproduce the $\pi^{-}$interaction length measured in the CHARM II detector exposed to a high-energy pion beam.

\subsection{Systematic uncertainty of background normalization}

After tuning the simulation with the samples of same-sign dimuons, the background induced in the opposite-sign dimuon samples can be calculated. The systematic uncertainty of the background normalization is composed of four contributions:

\footnotetext{
4) A global fit of the parameters was not performed and this is only a rough estimation of the errors taking into account the correlation between the parameters.

5) The required adjustment was achieved by increasing by $30 \%$ the default value $(0.3)$ of the LUND string fragmentation model's parameter defining the rate of the $s \bar{s}$ yield relative to the non-strange $q \bar{q}$ yield.
} 

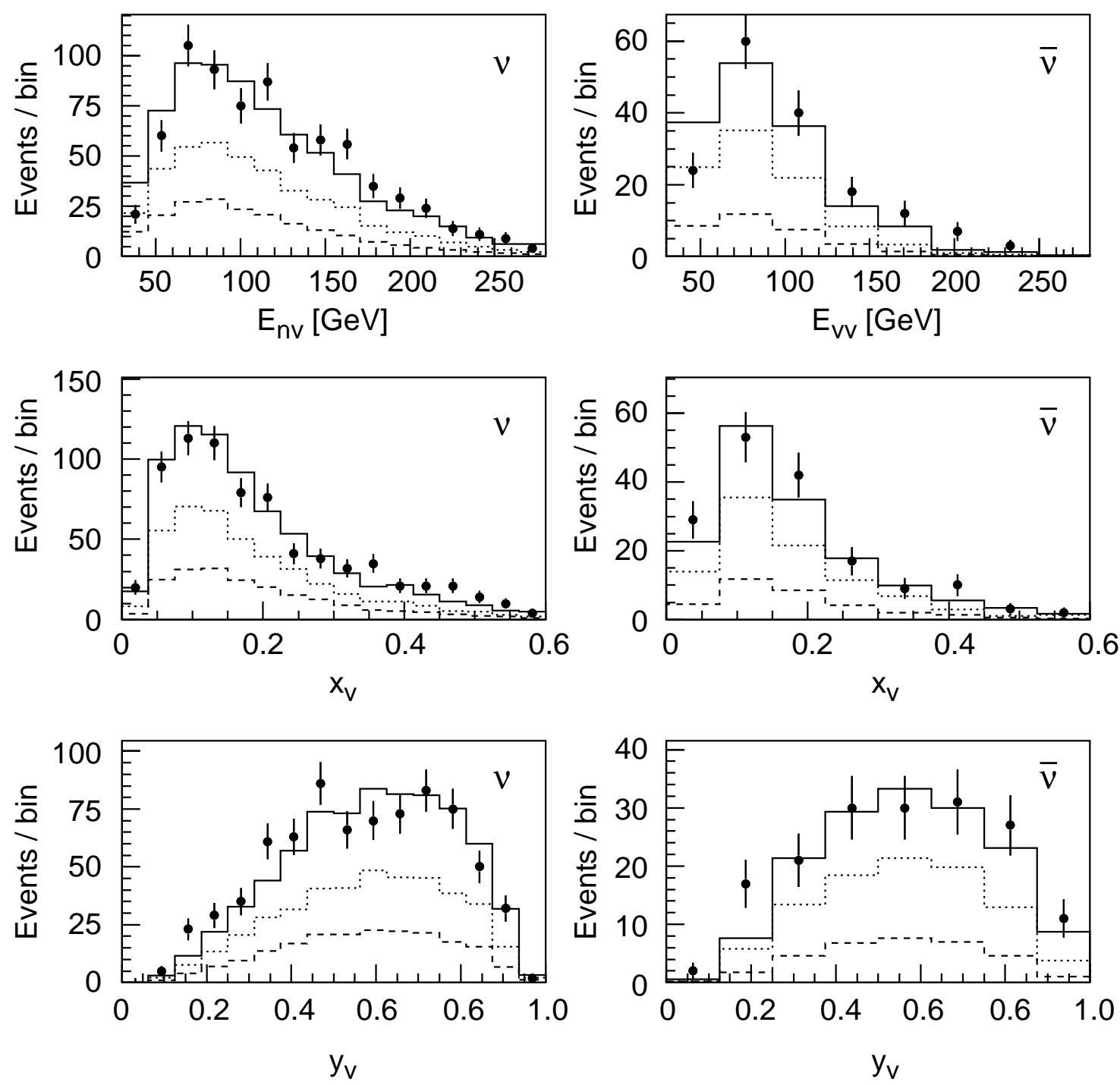

Figure 7: Comparison between distributions from the simulation (histograms) and the data (point) for neutrino- (left) and antineutrino- (right) induced same-sign dimuon events as a function of the (visible) neutrino energy $E_{\nu v}$, the (visible) Bjorken variables $x_{v}$ and $y_{v}$. In each figure, the displayed histograms represent from bottom to top the vertex pion, the vertex kaon, and the shower hadron contributions, respectively. 

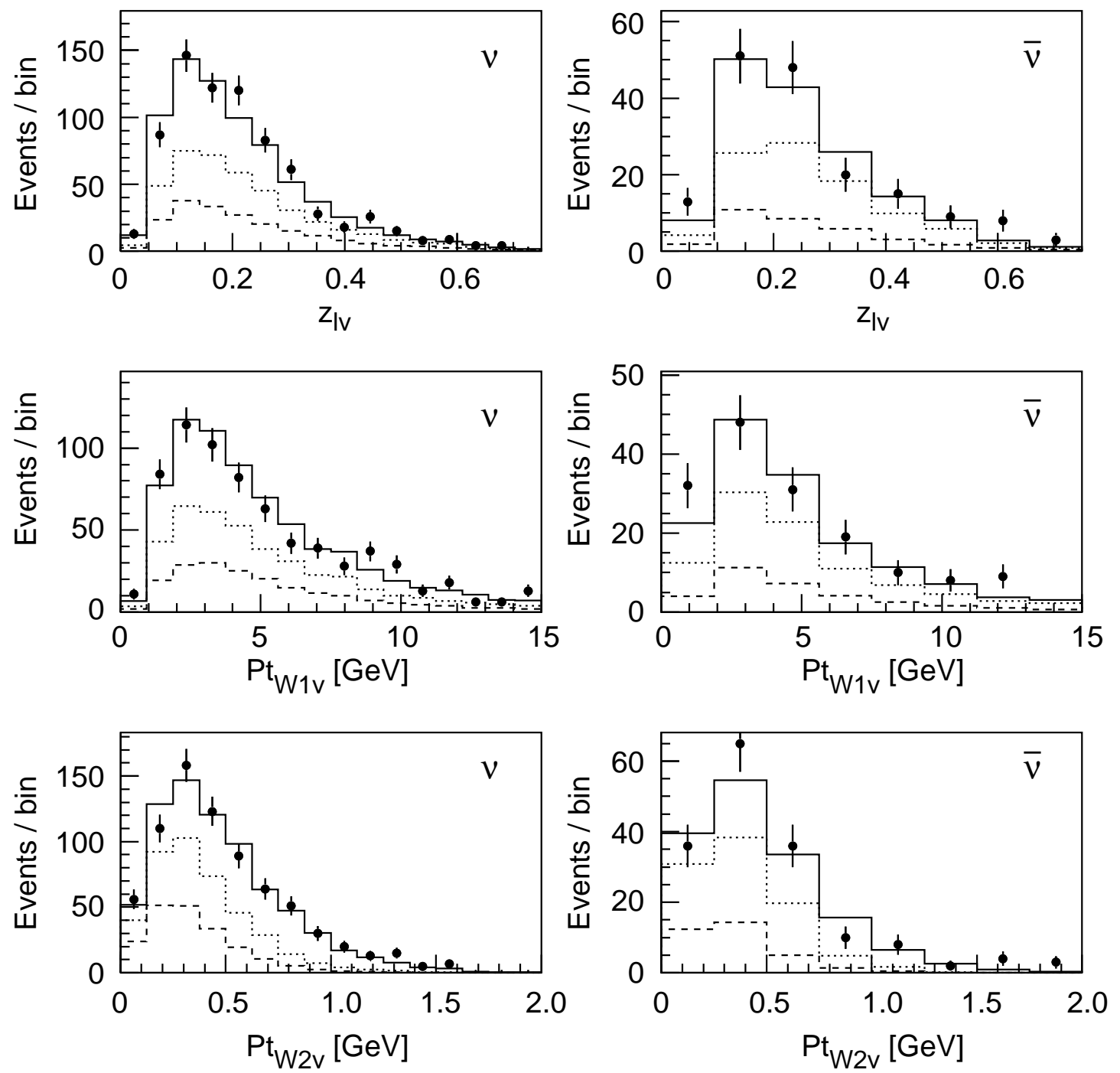

Figure 8: Comparison between the simulation (histograms) and the data (points) for neutrino- (left) and antineutrino- (right) induced same-sign dimuon events as a function of the $z_{l v}, P t_{w 1 v}$ and $P t_{w 2 v}$ distributions. 

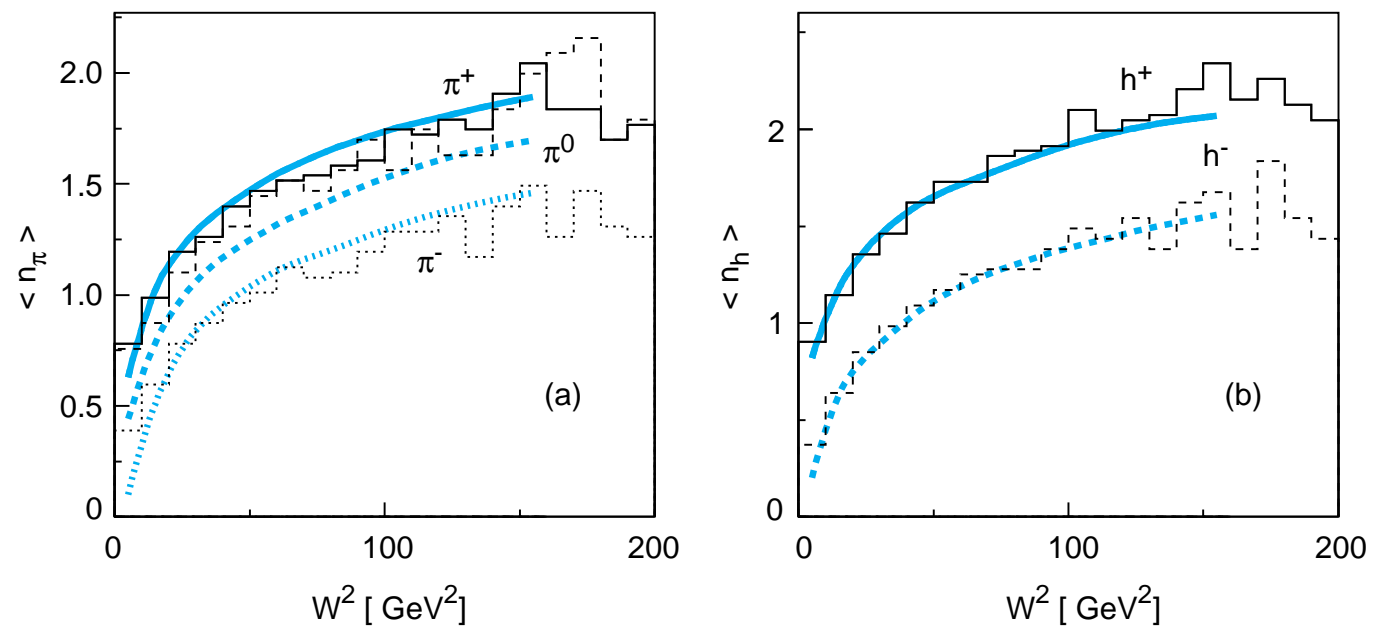

Figure 9: Mean number of pions (left) and charged hadrons (right) per charged current in the forward hemisphere as a function of the square of the hadronic invariant mass $W^{2}$.

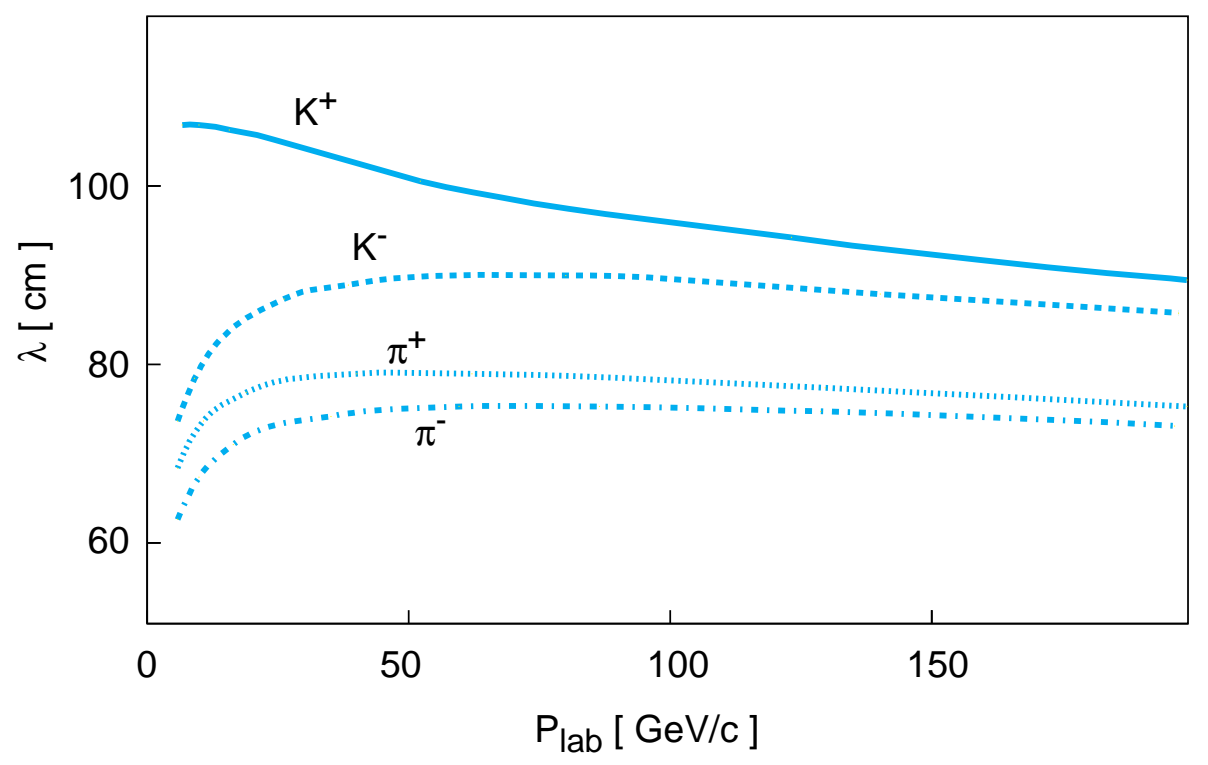

Figure 10: Interaction length for different mesons. 
- the uncertainty of the number of observed same-sign dimuon events;

- the error of the relative interaction lengths of $\pi^{+}\left(K^{+}\right)$and $\pi^{-}\left(K^{-}\right)$;

- the error of the relative multiplicities of mesons of opposite charge.

This and the previous terms arise from the application of the model tuned with the same-sign to the opposite-sign dimuons.

- The uncertainty of the relative spectrometer efficiency for the focused and unfocused low energetic muons.

The estimated values of these errors are given in Table 6 .

Table 6: Error sources of the opposite-sign background normalization

\begin{tabular}{|l|c|}
\hline Error sources & Relative error \\
\hline Normalization using same-sign & 0.08 \\
Relative interaction lengths & 0.04 \\
Relative multiplicities & 0.04 \\
Relative spectrometer efficiency & 0.10 \\
\hline Total error & 0.14 \\
\hline
\end{tabular}

\section{$6 \quad$ Analysis}

\section{1 $\quad$ Fitting procedure}

The five free parameters introduced in Section 2 are determined by fitting the observed differential event distributions to the model distributions obtained by a Monte Carlo simulation of dimuon events, with the beam properties, detector response and background processes properly taken into account as described in the previous sections. The normalization of the Monte Carlo dimuon sample is fixed by the requirement that the generated single-muon event rate per incident neutrino should match the known total rate of $\mathrm{CC}$ events induced in the detector (Section 3). The Monte Carlo sample consists of $25000 \nu$ - and $8800 \bar{\nu}$ - induced dimuon events, or $\sim 6$ and $\sim 10$ times the size of the corresponding data samples.

For fitting we use 2-dimensional $\left(x_{v}, E_{\nu v}\right)$ and 1-dimensional $z_{l v}$ distributions, which are the most sensitive to our set of free parameters. To obtain the model distributions, each simulated event is given a weight proportional to the cross-section (6), calculated with 'seed' values of the parameters. The data sample is split into 70 bins in $\left(x_{v}, E_{\nu v}\right)$ and 15 bins in $z_{l v}$, with the binning defined by the available statistics and resolutions (Appendix, Tables A1-A4). The parameters are determined by means of a $\chi^{2}$ minimization procedure performed in several iterations. At each step a new Monte Carlo sample is generated with the model parameters obtained at the previous step. At the last iteration, the starting parameter values were within 1-2 standard deviations from their final values and further re-fits yielded statistically compatible results. Table 7 shows the fit results, corresponding to a $\chi^{2}=99$ for 80 degrees of freedom. Figure 11 illustrates a strong correlation between $m_{c}$ and $\kappa$ (see also Table A5 in the Appendix).

In order to test the possible dependence of the results on the selection criteria, the fit was repeated for events within smaller $\left(x_{v}, E_{\nu v}\right)$ and $z_{l v}$ domains and/or with stricter cuts on other kinematical and topological variables, such as the hadronic energy $E_{\text {had }}, y_{v}$, $Q_{v}^{2}, r_{\text {spec }}$ (the radius of the muon impact point at the spectrometer entrance) and $N_{v x}$ (the 


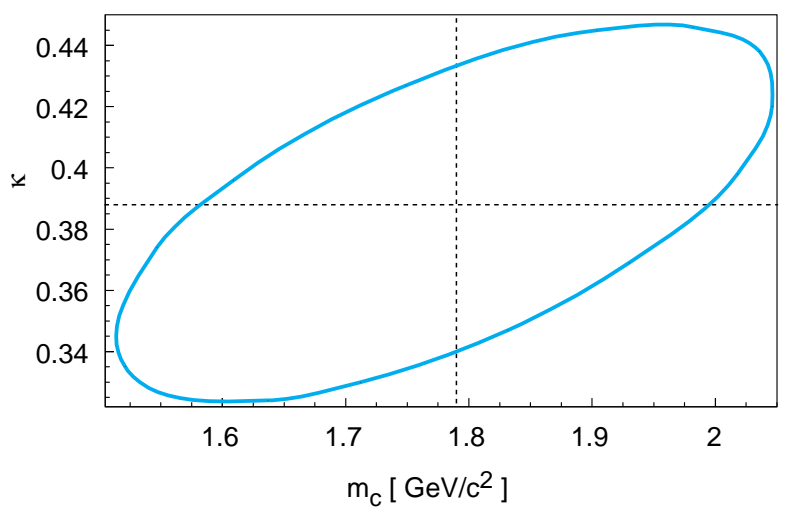

Figure 11: One standard deviation ellipse for the parameters $\kappa$ and $m_{c}$.
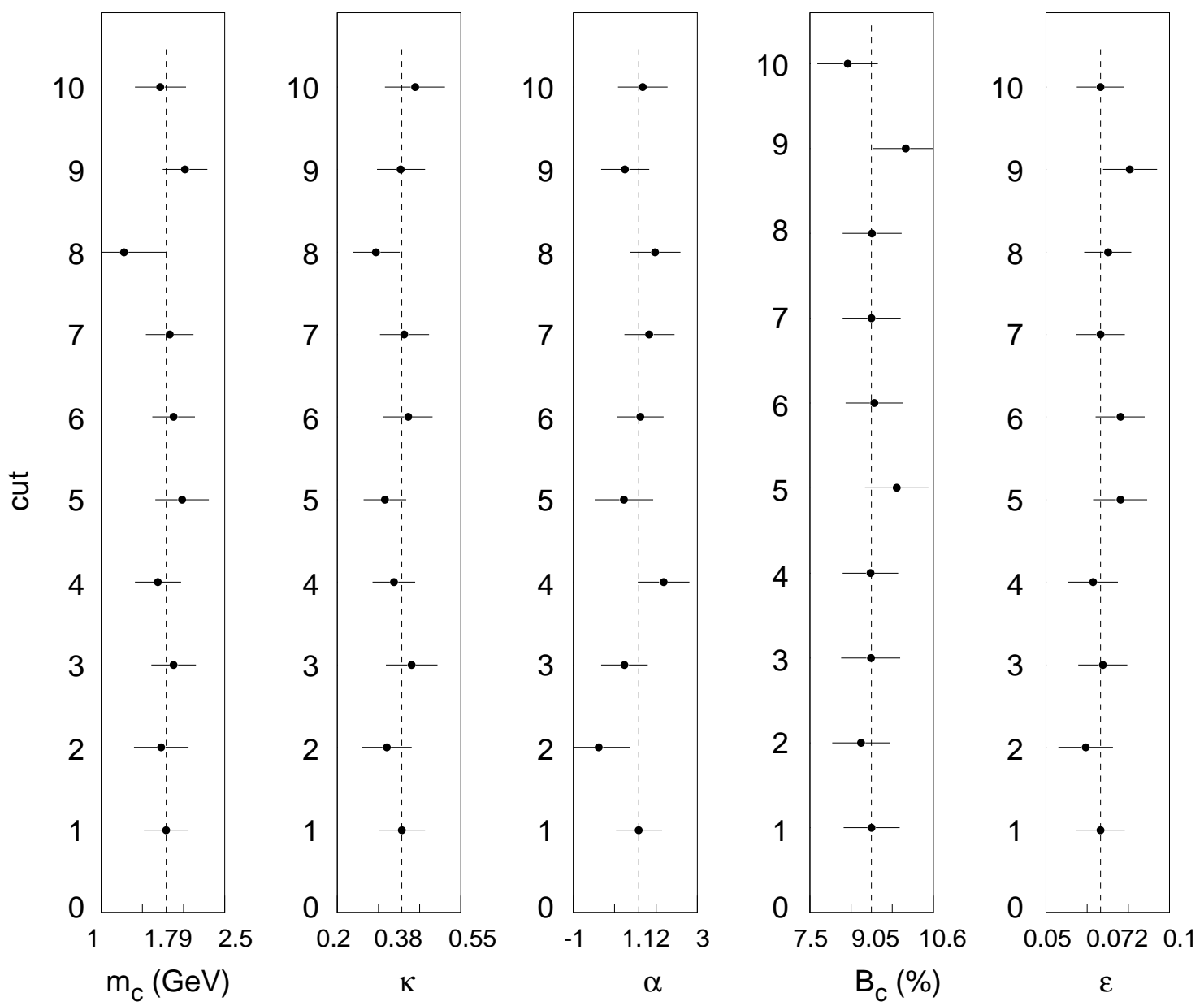

Figure 12: Fit results for different cuts: (1) default, (2) $x_{v}>0.1$, (3) $z_{l v}>0.15$, (4) $y_{v}>0.3$, (5) $Q_{v}^{2}>8 \mathrm{GeV}^{2}$, (6) $E_{\mathrm{had}}>10 \mathrm{GeV}$, (7) $E_{2}>8 \mathrm{GeV}$, (8) $E_{\nu v}>60 \mathrm{GeV}$, (9) $r_{\text {spec }}<150 \mathrm{~cm}$ (the radius of the muon impact point at the spectrometer entrance, $<190 \mathrm{~cm}$, by default), (10) $N_{v x}<360$. 
Table 7: Fit results for the free parameters of the dimuon cross-section. The errors are statistical.

\begin{tabular}{|c|c|c|c|c|}
\hline$m_{c}\left(\mathrm{GeV} / c^{2}\right)$ & $\kappa$ & $\alpha$ & $B_{\mu}(\%)$ & $\epsilon$ \\
\hline $1.79_{-0.28}^{+0.26}$ & $0.388_{-0.061}^{+0.074}$ & $1.12_{-0.72}^{+0.78}$ & $9.05_{-0.69}^{+0.71}$ & $0.072_{-0.009}^{+0.010}$ \\
\hline
\end{tabular}

vertex plane number). As shown in Fig. 12, this resulted in no significant variation of the fitted parameter values, which proves the robustness of the fitting procedure and of the model itself. However, the enhanced value of $\chi_{n d f}^{2} \simeq 1.2$ and the presence of systematic discrepancies in the shapes of the Monte Carlo calculated and observed distributions of relevant kinematical variables (Figures 13, 14 and 15) suggests that the model does not provide a fully adequate description of the data.

\subsection{Systematic uncertainties}

The systematic uncertainties of the result of the fit are mainly due to the theoretical model and its uncertainties, the fast detector response simulation, the background description, and the neutrino flux normalization.

To judge the impact of the quark distribution functions and the charm fragmentation function, we tried the GRV [29] and CCFR [30] quark distribution functions sets, with the Collins-Spiller fragmentation function [31] in a separate fit. The resulting variation in the fit parameters is assumed to be one constituent of the theoretical error ${ }^{6}$ ). Another one is due to parameters in the dimuon cross-section which are known with a finite accuracy (like the slope parameter of the $p_{t}$ distribution or semileptonic branching fractions). The corresponding contributions to systematic errors are obtained by varying these parameters within their errors. A similar approach is used to evaluate the contributions due to uncertainties in the neutrino flux normalization and the background description.

The uncertainties related to the fast detector response simulation originate from the determination of the spectrometer efficiency for small muon momenta, the simulation of the dimuon trigger efficiency (especially the trigger losses due to muon tracks hidden in the shower), and from hadronic and muon energy scale calibration. The corresponding systematic errors are estimated in the following way. The difference between the experimental distribution of a given quantity (such as $E_{\text {had }}, E_{\mu}^{\text {spec }}, N_{v x}$, etc.) and the corresponding Monte Carlo distribution obtained with the best fit parameters is assumed to be entirely due to a systematic error of the detector response. The efficiencies and the energy scales are then adjusted in such a way that the best possible match with the Monte Carlo is obtained, then a new fit is performed, and the differences between the old and new fits are regarded as the corresponding contributions to the systematic errors.

The uncertainties due to neutrino flux and background normalization were obtained by a propagation of the corresponding errors quoted in Sections 3.2 and 5.3. The influence of the background shape on the fitted parameters was assessed by switching off alternatively each of the background sources (vertex and shower hadrons) and renormalizing the remaining sources to the entire same-sign sample.

A summary of the systematic errors is given in Table 8 .

\footnotetext{
6) This does not apply to $\epsilon$, which is not identical in the Collins-Spiller and Peterson parametrizations.
} 

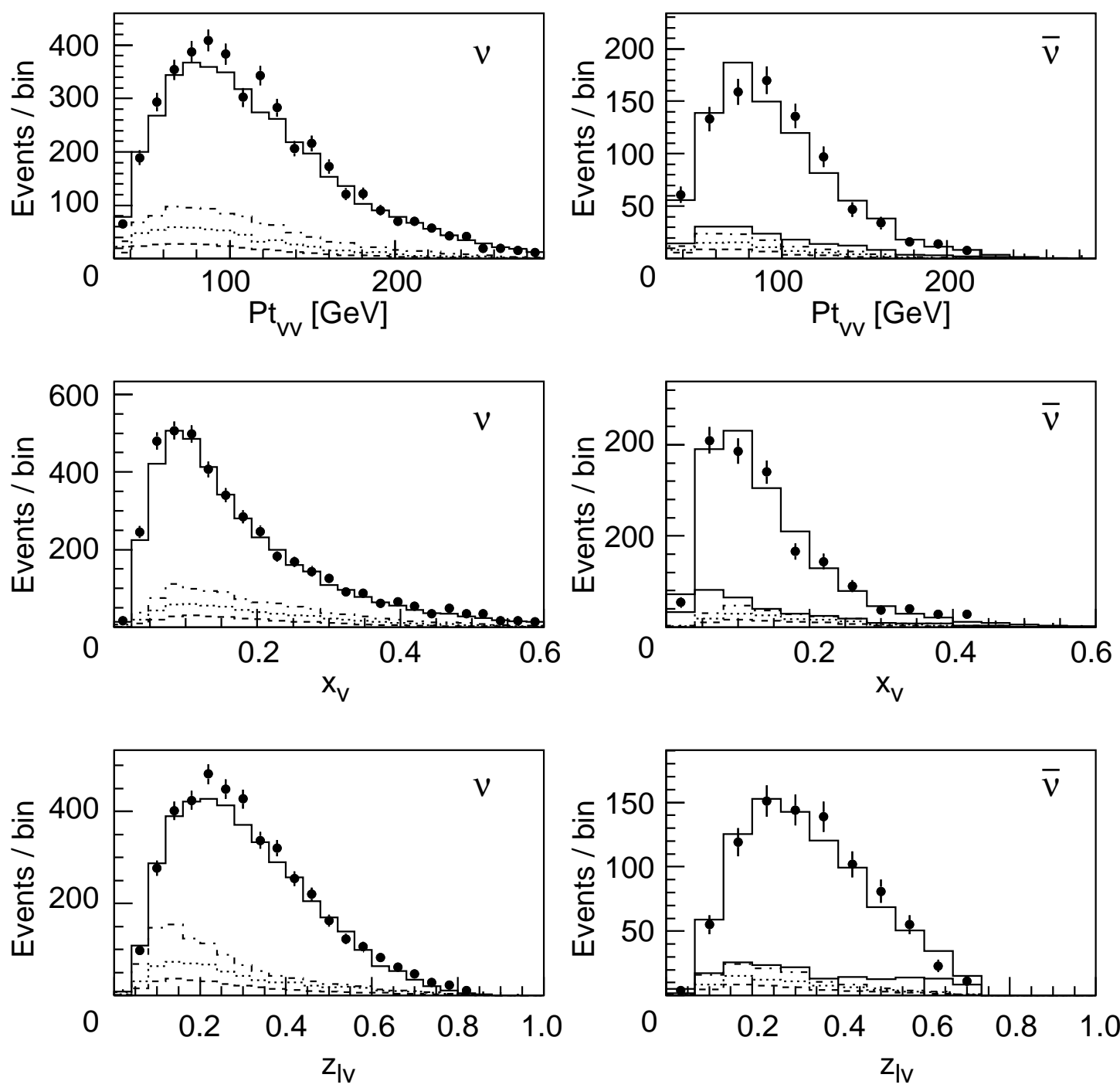

Figure 13: Comparison between simulated (histograms) and experimental (points) distributions of the visible neutrino energy $E_{\nu v}$, visible Bjorken variable $x_{v}$, and $z_{l v}$ variable, for neutrino- and antineutrino-induced dimuon events. In all plots, contributions from vertex pions, vertex kaons, and shower hadrons are shown as stacked on top of each other, from bottom to top, respectively. In antineutrino plots, an additional contribution from the $\nu_{\mu}$ contamination in the beam is also shown. 

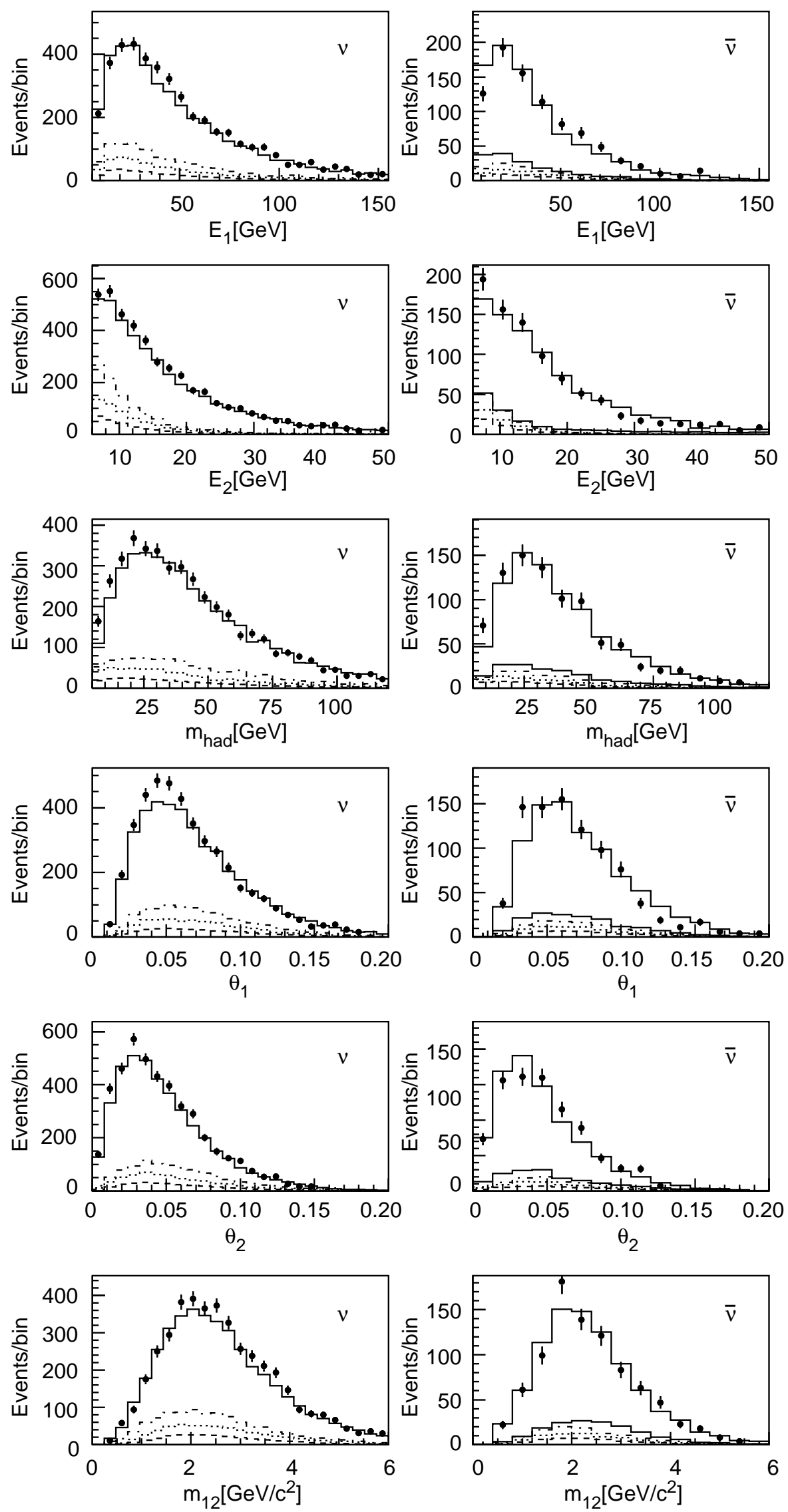

Figure 14: Same as Fig. 13, for the variables not used for fitting: muon energies, $E_{1}$ and $E_{2}$, the hadronic energy $E_{\text {had }}$, muon track angles with respect to the neutrino beam, $\theta_{1}$ and $\theta_{2}$, and the muon invariant mass $m_{12}$. 

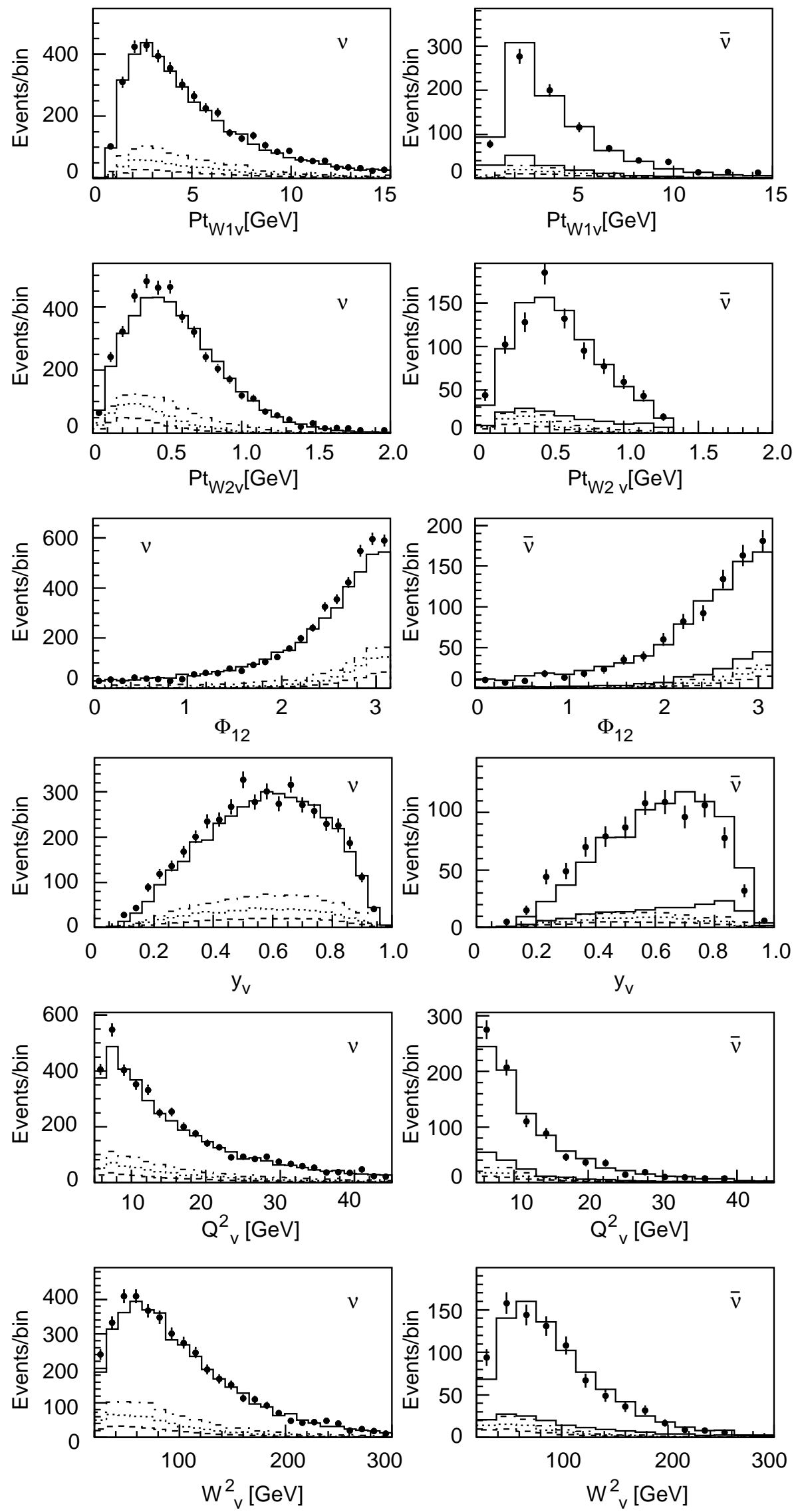

Figure 15: Same as Fig. 14, for $P t_{w 1 v}, P t_{w 2 v}, \phi_{12}, y_{v}, Q_{v}^{2}$ and $W_{v}^{2}$. 


\subsection{Fit results without assuming $\left|V_{c d}\right|$ and $\left|V_{c s}\right|$ values}

The part of the dimuon cross section sensitive to parton densities can be expressed as

$$
\frac{d^{3} \sigma^{\nu(\bar{\nu}) \rightarrow 2 \mu}}{d x d y d z} \propto\left|V_{c d}\right|^{2} B_{\mu}\left[\left(d_{v}\right)+\frac{1}{2+A_{s}(1-x)^{\alpha}}\left(1+\left|\frac{V_{c s}}{V_{c d}}\right|^{2} A_{s}(1-x)^{\alpha}\right) \bar{q}\right]
$$

where the valence quark contribution $d_{v}=0$ for $\bar{\nu}$. This expression shows explicitly that the fitted value of $B_{\mu}$ is correlated with the assumed value of $\left|V_{c d}\right|^{2}$. The result of the fit can also be presented as the product of the two parameters $\left|V_{c d}\right|^{2} B_{\mu}$. The strange quark density is then multiplied by the ratio $\left|V_{c s} / V_{c d}\right|^{2}$.

A new five-parameter fit, with the yield factor $\left|V_{c d}\right|^{2} B_{\mu}$ fitted instead of $B_{\mu}$, has been performed for different values of $\left|V_{c s} / V_{c d}\right|$. The only parameter which changed significantly is $\kappa$. The values of $m_{c}, \epsilon$ and $\left|V_{c d}\right|^{2} B_{\mu}$ remain practically the same. Table 9 shows the maximum variation of the fitted parameters for a $\left|V_{c s} / V_{c d}\right|$ variation of up to $20 \%$ from the default value.

Table 8: Dominant systematic uncertainties of fitted parameter values

\begin{tabular}{|l|c|c|c|c|c|}
\hline Error sources & $\Delta m_{c}$ & $\Delta \kappa$ & $\Delta \alpha$ & $\Delta B_{\mu}(\%)$ & $\Delta \epsilon$ \\
\hline \hline Structure function & 0.07 & 0.030 & 1.00 & 0.40 & 0.003 \\
Fragmentation & 0.03 & 0.003 & 0.04 & 0.00 & \\
$p_{t}$ distribution & 0.10 & 0.018 & 0.15 & 0.06 & 0.002 \\
Decay $K^{*}$ versus $K$ & 0.06 & 0.020 & 0.09 & 0.02 & 0.002 \\
\hline$\left|V_{c d}\right|$ and $\left|V_{c s}\right|$ & 0.00 & 0.013 & 0.00 & 0.25 & 0.000 \\
\hline Spectrometer efficiency & 0.12 & 0.030 & 0.10 & 0.10 & 0.004 \\
Trigger simulation & 0.06 & 0.020 & 0.07 & 0.01 & 0.000 \\
Energy scale & 0.15 & 0.030 & 0.05 & 0.05 & 0.010 \\
\hline Background normalization & 0.03 & 0.005 & 0.006 & 0.31 & 0.008 \\
Background shape & 0.10 & 0.015 & 0.005 & 0.22 & 0.004 \\
\hline Neutrino flux & 0.00 & 0.000 & 0.000 & 0.36 & 0.000 \\
Relative flux & 0.02 & 0.014 & 0.070 & 0.18 & 0.000 \\
\hline \hline Total & 0.27 & 0.067 & 1.027 & 0.74 & 0.015 \\
\hline
\end{tabular}

Table 9: Maximum relative variation of parameters for a variation of $\left|V_{c s} / V_{c d}\right|$ of up to $20 \%$

\begin{tabular}{|l|c|c|c|c|c|}
\hline Parameter & $m_{c}$ & $\kappa$ & $\alpha$ & $\left|V_{c d}\right|^{2} B_{\mu}$ & $\epsilon$ \\
\hline Maximum variation in $\%$ & 0.3 & 76.5 & 7.8 & $<0.01$ & 0.02 \\
\hline
\end{tabular}

The fit result for the dimuon 'yield' factor of the cross-section is:

$$
\left|V_{c d}\right|^{2} B_{\mu}=\left(4.42_{-0.34}^{+0.35} \pm 0.34\right) \times 10^{-3} .
$$

For $\alpha=0$, the sea contribution to the cross-section is proportional to $\frac{\left(1+\left|V_{c s} / V_{c d}\right|^{2} \kappa\right)}{(2+\kappa)}$. This quantity is practically insensitive to the variations of $\left|V_{c s} / V_{c d}\right|$ and within $0.01 \%$ remains equal to 


$$
\frac{1}{2+\kappa}\left(1+\left|\frac{V_{c s}}{V_{c d}}\right|^{2} \kappa\right)=3.58_{-0.41}^{+0.49} \pm 0.44,
$$

with the errors derived from the uncertainty of $\kappa$. Relations (8) and (9) can be used to extract parameters $\left|V_{c d}\right|$ and $\left|V_{c s}\right|$ from an independent determination of $B_{\mu}$ and $\kappa$.

\subsection{Model variations}

\subsubsection{A violation of the Callan-Gross relation}

In Eq. (1), the Callan-Gross relation holds in terms of the rescaling variable $\chi$

$$
F_{2}(\chi)=2 \chi F_{1}(\chi)
$$

The modified leading-order analysis of CCFR [12] takes into account the violation of the Callan-Gross relation rather arbitrarily by introducing the longitudinal structure function $R_{L}$ as follows:

$$
F_{2}(\chi)=\frac{1+R_{L}\left(\chi, Q^{2}\right)}{1+(2 M \chi / Q)^{2}} 2 \chi F_{1}(\chi)
$$

which corresponds to multiplying the cross-section (1) by the factor [32]

$$
\frac{\frac{1+R_{L}\left(\chi, Q^{2}\right)}{1+(2 M \chi / Q)^{2}}(1-y)+\frac{x y}{\chi}}{1-y+\frac{x y}{\chi}}
$$

using external measurements of the structure function $R_{L}\left(\chi, Q^{2}\right)$ [33].

The relative changes in the fit results obtained with this model modification are shown in Table 10. The corrections are smaller than the corresponding parameter errors in the main fit (Table 7).

Table 10: Relative changes of the parameters following the CCFR prescription to take into account the violation of the Callan-Gross relation

\begin{tabular}{|l|c|c|c|c|c|}
\hline Parameter & $m_{c}$ & $\kappa$ & $\alpha$ & $\left|V_{c d}\right|^{2} B_{\mu}$ & $\epsilon$ \\
\hline Variation in $\%$ & -2.2 & -5.5 & -14.4 & -3.1 & +2.9 \\
\hline
\end{tabular}

\subsubsection{Fit results without QED radiative correction}

The radiative correction factor $\left(1+\delta_{r}\right)$ is calculated for the inclusive charged current cross-section and is assumed to be the same for the dimuon cross-section. In order to see the effects of a possible violation of this hypothesis, a new fit is performed with $\delta_{r}=0$, and the relative changes of the parameters are shown in Table 11. Parameters $m_{c}$ and $\left|V_{c d}\right|^{2} B_{\mu}$ exhibit a remarkable stability. This can be explained by the fact that they rely mainly on the energy dependence of the cross-section (after integration on $x$ and $y$ ) which is almost unaffected by the radiative correction. On the contrary, the expected migration of events from small $y$ to high $y$ (or, for fixed values of $Q^{2}$ and $E_{\nu}$, from high $x$ to small $x)$ can explain qualitatively the expected smaller value for $\kappa$. 
Table 11: Relative changes of the parameters assuming no radiative corrections $\left(\delta_{r}=0\right)$.

\begin{tabular}{|l|c|c|c|c|c|}
\hline Parameter & $m_{c}$ & $\kappa$ & $\alpha$ & $\left|V_{c d}\right|^{2} B_{\mu}$ & $\epsilon$ \\
\hline variation in $\%$ & -1.9 & -15.0 & +36.7 & +1.5 & +0.2 \\
\hline
\end{tabular}

\subsection{Summary of the analysis results}

On the basis of a leading-order calculation of the cross-section for opposite-sign dimuon production by neutrinos and antineutrinos, we have obtained the following values for the charm quark mass $m_{c}$, parameters $\kappa$ and $\alpha$ of the nucleon strange quark distribution, the parameter $\epsilon$ of the Peterson fragmentation function and the product of the square of the CKM mixing matrix element $\left|V_{c d}\right|$ and the average semi-muonic branching ratio of charm hadrons $B_{\mu}$ :

$$
\begin{array}{cl}
m_{c} & =\left(1.79_{-0.28}^{+0.26} \pm 0.27\right) \mathrm{GeV} / c^{2} \\
\kappa & =0.388_{-0.061}^{+0.074} \pm 0.067 \\
\alpha & =1.12_{-0.72}^{+0.78} \pm 1.03 \\
\epsilon & =0.072_{-0.009}^{+0.010} \pm 0.015 \\
\left|V_{c d}\right|^{2} B_{\mu} & =\left(4.42_{-0.34}^{+0.35} \pm 0.34\right) \times 10^{-3}
\end{array}
$$

where the first error is statistical and the second is systematic.

The variations observed under different assumptions for $R_{L}$ and $\delta_{r}$, as discussed in Sections 6.4.1 and 6.4.2, are not included in the systematic error. These variations should rather be attributed to the choice of the model.

Using the independent measurement of $\bar{B}_{\mu}(5)$, we determine the value of $\left|V_{c d}\right|$ :

$$
\left|V_{c d}\right|=0.219 \pm 0.012 \pm 0.011
$$

where the first and the second error come from the uncertainty of $\left|V_{c d}\right|^{2} B_{\mu}$ and $\bar{B}_{\mu}$, respectively.

The strange content of the nucleon can also be presented in terms of the $\eta$ parameter defined as the ratio of the strange sea relative to the total up and down quarks, $\eta=$ $2 S /(U+D)$. Assuming $\bar{Q} / Q=0.203$ from CTEQ parametrization ${ }^{7)}$, we obtain

$$
\eta=0.068 \pm 0.014
$$

where the error combines statistical and systematic uncertainties.

\section{$7 \quad$ Discussion}

A comparison of our results with those reported by the CDHS [16] and CCFR [12] collaborations is shown in Table 12. All measured parameters, except $\epsilon$ (or $\langle z\rangle$ ), are compatible within the errors.

The positive value of $\alpha$ qualitatively confirms the conclusion of CCFR that the strange sea is softer than the non-strange sea. However, the large uncertainty of this value

\footnotetext{
7) The error on $\bar{Q} / Q$ is implicitly taken into account in the systematic errors due to the choice of parton
} densities. 
and its strong model dependence indicate that within the framework of this analysis $\alpha$ should be regarded as a tuning rather than a physics parameter.

The notable discrepancy between the mean values of the charm quark momentum fraction $\langle z\rangle$ taken by the charm particle, determined by CHARM II and CCFR using the same method, can be interpreted as an indication of the energy dependence of this parameter. On the other hand, the agreement of the measured (CDHS) and derived (CHARM II) values of $\langle z\rangle$, obtained for the same neutrino beam, justifies the use of the Peterson fragmentation model.

Table 12: Comparison of CHARM II, CHDS and CCFR LO analysis results. Statistical and systematic errors added in quadrature. For CHARM II and CCFR, $\langle z\rangle$ is calculated from (4) using the fitted value of $\epsilon$. For CDHS, the range for $\epsilon$ is obtained from the direct determination of $\langle z\rangle$. All these derived numbers are given in parentheses.

\begin{tabular}{|c|c|c|c|}
\hline & CHARM II & CDHS & CCFR \\
\hline \hline & Number of observed dimuon events less the background \\
\hline \hline$N_{2 \mu}, \quad \nu$ & $\sim 3100$ & $\sim 8600$ & $\sim 4200$ \\
\hline$N_{2 \mu}, \quad \bar{\nu}$ & $\sim 700$ & $\sim 2000$ & $\sim 900$ \\
\hline \hline & \multicolumn{3}{|c|}{ Charm quark mass (in GeV $\left./ c^{2}\right)$} \\
\hline \hline$m_{c}$ & $1.79 \pm 0.38$ & $1.50 \pm 0.30$ & $1.31 \pm 0.23$ \\
\hline \hline & \multicolumn{3}{|c|}{ Strange quark content parameters } \\
\hline \hline$\kappa$ & $0.39 \pm 0.09$ & $0.48 \pm 0.08$ & $0.37 \pm 0.05$ \\
\hline$\eta$ & $0.068 \pm 0.014$ & $0.061 \pm 0.005$ & $0.064 \pm 0.008$ \\
\hline$\alpha$ & $1.12 \pm 1.29$ & 0 (fixed) & $2.5 \pm 0.65$ \\
\hline \hline & \multicolumn{3}{|c|}{ Fragmentation parameters } \\
\hline \hline$\epsilon$ & $0.072 \pm 0.017$ & $([0.02,0.14])$ & $0.22 \pm 0.05$ \\
\hline$\langle z\rangle$ & $(0.66 \pm 0.03)$ & $0.68 \pm 0.08$ & $(0.56 \pm 0.03)$ \\
\hline \hline & $4.42 \pm 0.48$ & $4.10 \pm 0.72$ & $5.09 \pm 0.36$ \\
\hline \hline$\left|V_{c d}\right|^{2} B_{\mu} \times 10^{3}$ & $4.4 m u o n$ yield factor \\
\hline
\end{tabular}

As a useful graphical illustration of the model cross-section (1), Fig. 16 shows dimuon to single muon cross-section ratios of $\nu_{\mu}$ and $\bar{\nu}_{\mu}$ as functions of energy, calculated with parameters from the three experiments, for the kinematical domain $Q^{2}>$ $6 \mathrm{GeV}$ and $\nu>15 \mathrm{GeV}$, approximately covering our data sample for $E_{\nu}>35 \mathrm{GeV}$. 

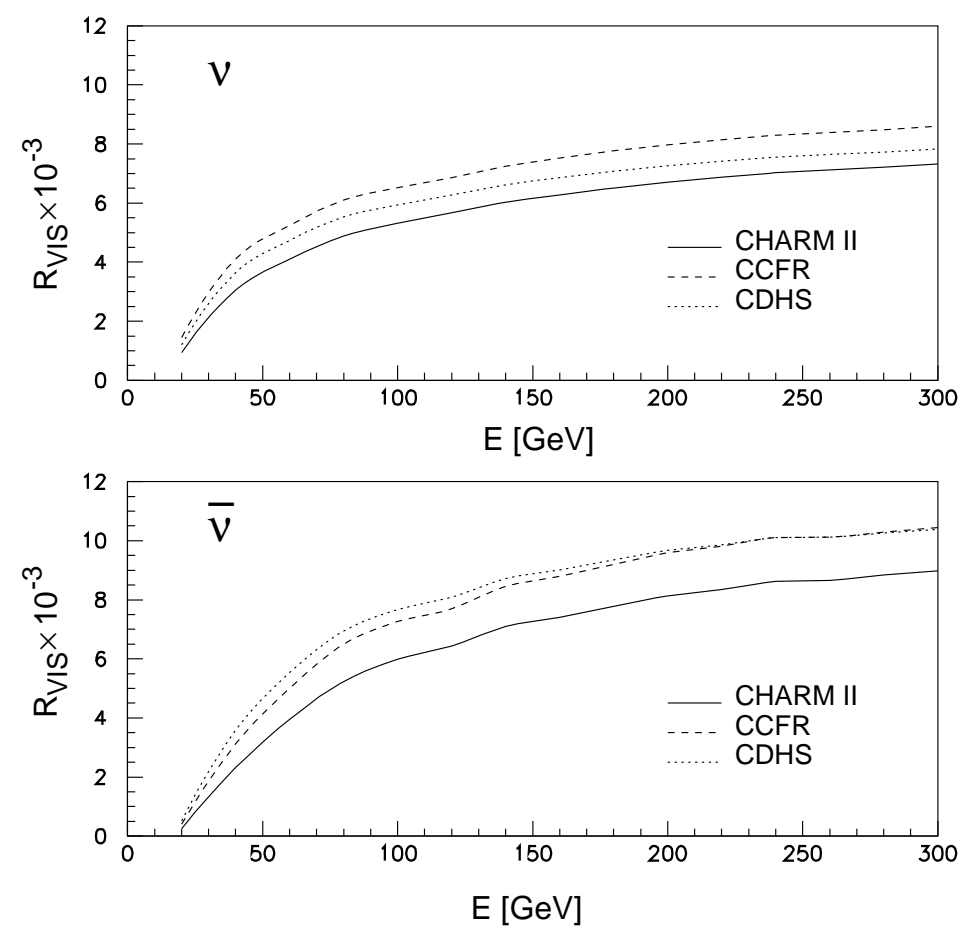

Figure 16: Opposite-sign dimuon to single muon cross-section ratio for $Q^{2}>6 \mathrm{GeV}^{2}$ and $\nu>15 \mathrm{GeV}$ for neutrinos and antineutrinos. The curves correspond to the crosssection ratios calculated with the parameters resulting from CHARM II, CCFR and CDHS analyses.

The physical interpretation of parameters $m_{c}$ and $\kappa$ as the charm quark mass and the strange content of the nucleon requires some caution, notwithstanding their stability and a good description of our data by the LO formalism. Recent next-to-leading order (NLO) analysis of dimuon data [32] showed that these parameters are sensitive to a gluon fusion contribution, particularly near the reaction threshold.

Nonetheless, $m_{c}$ determined in this analysis is adequate for defining a more accurate value of $\sin ^{2} \theta_{W}$ from the ratio of neutral-current to charged-current deep-inelastic crosssections. Table 13 quotes the results of $\sin ^{2} \theta_{W}$ measurement by CDHS [34], CHARM [35] and CCFR [36], parametrized as linear functions of $m_{c}{ }^{8)}$.

Table 13: Experimental values for $\sin ^{2} \theta_{W}$ from CDHS [34], CHARM [35] and CCFR [36] collaborations, evaluated at $m_{\text {top }}=175 \mathrm{GeV} / c^{2}$ and $M_{\text {Higgs }}=150 \mathrm{GeV} / c^{2}$. The experimental error includes both systematic and statistical errors. The theoretical error does not include the dependence on the charm quark mass $\left(m_{c}\right)$, which is shown explicitly.

\begin{tabular}{|l|c|c|c|c|}
\hline Experiment & $\sin ^{2} \theta_{W}$ & $m_{c}$ dependence & $\Delta_{\exp }$ & $\Delta_{\text {theo }}$ \\
\hline CHARM & 0.2343 & $0.012\left(m_{c}-1.50\right)$ & 0.0051 & 0.0024 \\
CDHS & 0.2257 & $0.013\left(m_{c}-1.50\right)$ & 0.0054 & 0.0024 \\
CCFR & 0.2236 & $0.011\left(m_{c}-1.31\right)$ & 0.0027 & 0.0018 \\
\hline
\end{tabular}

8) The quoted values are corrected using the latest re-evaluation of the radiative corrections for $m_{\text {top }}=$ $175 \mathrm{GeV} / c^{2}$ and $M_{\mathrm{Higgs}}=150 \mathrm{GeV} / c^{2}$ [37]. The original values from CHARM and CDHS, obtained for $m_{\mathrm{top}}=45 \mathrm{GeV} / c^{2}$ and $m_{\mathrm{top}}=60 \mathrm{GeV} / c^{2}$ and for $M_{\mathrm{Higgs}}=100 \mathrm{GeV} / c^{2}$, respectively, have been decreased by 0.0017 and 0.0027 , respectively. 
Using the average of the three measurements of $m_{c}$ from Table $12,\left\langle m_{c}\right\rangle=(1.46 \pm$ $0.17) \mathrm{GeV} / c^{2}$, we obtain a new estimate of the electroweak parameter

$$
\sin ^{2} \theta_{W}=0.2268 \pm 0.0022(\exp ) \pm 0.0028 \text { (theor) }
$$

where the theoretical error includes the uncertainty caused by the charm quark mass.

The quoted value of $\sin ^{2} \theta_{W}$ is defined in the Sirlin (on-shell) renormalization scheme, and can therefore be translated to the $W$-boson mass using the high precision measurement of the $Z$-boson mass $M_{Z}=(91.1867 \pm 0.0020) \mathrm{GeV} / c^{2}$ from LEP experiments [38]:

$$
M_{W}^{\nu}=(80.18 \pm 0.18) \mathrm{GeV} / c^{2} .
$$

This result is compatible with the direct measurements $M_{W}^{\nu}=(80.43 \pm 0.08) \mathrm{GeV} / c^{2}$ from the observation of $W$ at CERN and Fermilab [38].

A more precise value of $\left|V_{c d}\right|$ can be obtained by averaging the measured factors $\left|V_{c d}\right|^{2} B_{\mu}$ from Table $12\left[\left\langle\left|V_{c d}\right|^{2} B_{\mu}\right\rangle=(4.75 \pm 0.27) \times 10^{-3}\right]$ and using the updated average semi-muonic branching ratio of charm hadrons (5). We obtain

$$
\left|V_{c d}\right|=0.227 \pm 0.006 \pm 0.012
$$

where the first error comes from the uncertainty on $\left|V_{c d}\right|^{2} B_{\mu}$ and the second error from $B_{\mu}$. This value of $\left|V_{c d}\right|$ is in good agreement with the value of $\left|V_{c d}\right|=0.221 \pm 0.003$ obtained by assuming unitarity of CKM mixing matrix and using the experimental values for other matrix elements from Ref. [9].

\section{Summary}

In the framework of a leading-order QCD formalism, the charm quark mass $m_{c}=$ $1.79 \pm 0.38 \mathrm{GeV} / c^{2}$, the strange content of the nucleon $\eta=0.068 \pm 0.014$ and the dimuon yield factor $\left|V_{c d}\right|^{2} B_{\mu}=(4.42 \pm 0.48) \times 10^{-3}$ have been determined and proved to be compatible with results of previous leading-order QCD analyses of dimuon production by neutrinos.

Assuming the PDG value for $\left|V_{c s} / V_{c d}\right|$, the data indicates that the strange quark content of the nucleon is suppressed with respect to non-strange sea quarks by a factor $\kappa=0.39 \pm 0.09$ and is somewhat softer than the non-strange sea at $\left\langle Q^{2}\right\rangle \simeq 15 \mathrm{GeV}^{2}$.

A combination of $\left|V_{c s} / V_{c d}\right|$ and $\kappa,\left(1+\left|V_{c s} / V_{c d}\right|^{2} \kappa\right) /(\kappa+2)=3.58 \pm 0.61$, is shown to be independent of the $\left|V_{c s} / V_{c d}\right|$ value.

By combining our results with those of previous similar analyses of dimuon events, new 'world average' values of the charm quark mass, $\left\langle m_{c}\right\rangle=(1.46 \pm 0.17) \mathrm{GeV} / c^{2}$, and the product of the square of the CKM mixing matrix element $\left|V_{c d}\right|$ and the average semimuonic branching ratio of charm hadrons $B_{\mu},\left\langle\left|V_{c d}\right|^{2} B_{\mu}\right\rangle=(4.75 \pm 0.27) \times 10^{-3}$, are obtained. From these numbers, new average values of $\sin ^{2} \theta_{W}$ and $\left|V_{c d}\right|$ are derived.

\section{Acknowledgements}

We gratefully acknowledge the help of our technical collaborators who contributed to the realization and the operation of the detector. We are grateful for the grants from the Inter-University Institute for Nuclear Sciences (Belgium), CERN (Geneva, Switzerland), the Bundesministerium für Forschung und Technologie (Germany), the Institute of Theoretical and Experimental Physics (Moscow, Russian Federation), the Istituto Nazionale di Fisica Nucleare (Italy), and the Turkish Scientific and Technical Research Council 
(TUBITAK), which made the experiment possible. Finally, we thank the CERN SPS operating crew and the neutrino beam staff for their competent assistance ensuring the excellent performance of their facilities.

\section{Appendix: data tables}

Tables A1, A2, A3 and A4 describe the data point attributes: number of events, errors, and the corresponding contribution to the $\chi^{2}$. The notations in these tables are:

$d:$ data

$t:$ theoretical prediction (signal)

$b$ : background

st : the squared statistical error for the signal prediction

$s b$ : the squared statistical error for the background

$\chi^{2}=(d-t-b)^{2} /(d+s t+s b)$

For $z_{l}$-points (Tables A3 and A4), the $\chi^{2}$ is calculated with the Monte Carlo distributions normalized to the data, to enhance the fit sensitivity to the shape parameter $\epsilon$ of the phenomenological fragmentation function. The sums of the contributions of $\left(x, E_{\nu}\right)$-points, $\left(x, E_{\bar{\nu}}\right)$-points, $z_{l}(\nu)$-points and $z_{l}(\bar{\nu})$-points to the overall $\chi^{2}=99$ are $63,22,11$ and 3 , respectively.

Table A5 shows the correlation matrix of the fitted parameters. 
Table A1: $\left(x, E_{\nu}\right)$ distribution (50 data points).

\begin{tabular}{|c|c|c|c|c|c|c|}
\hline$x:$ & $0-0.10$ & $0.10-0.15$ & $0.15-0.20$ & $0.20-0.30$ & $0.30-0.50$ & $E_{\nu}$ \\
\hline$d$ & 47.00 & 109.00 & 122.00 & 117.00 & 79.00 & \multirow{6}{*}{$35-60$} \\
\hline$t$ & 38.95 & 97.50 & 71.97 & 77.12 & 46.59 & \\
\hline$b$ & 12.21 & 35.32 & 33.11 & 51.74 & 33.07 & \\
\hline st & 9.96 & 21.55 & 12.57 & 9.81 & 3.88 & \\
\hline$s b$ & 2.16 & 4.50 & 4.56 & 8.99 & 6.54 & \\
\hline$\chi^{2}$ & 0.29 & 4.20 & 2.06 & 1.04 & 0.01 & \\
\hline$d$ & 58.00 & 88.00 & 51.00 & 76.00 & 58.00 & \multirow{6}{*}{$60-70$} \\
\hline$t$ & 54.24 & 68.43 & 38.42 & 43.75 & 24.38 & \\
\hline$b$ & 16.54 & 20.61 & 17.30 & 21.60 & 15.23 & \\
\hline st & 15.03 & 16.06 & 7.19 & 6.49 & 2.22 & \\
\hline$s b$ & 4.34 & 2.67 & 3.34 & 6.06 & 2.10 & \\
\hline$\chi^{2}$ & 2.11 & 0.01 & 0.36 & 1.28 & 5.43 & \\
\hline$d$ & 108.00 & 90.00 & 65.00 & 51.00 & 40.00 & \multirow{6}{*}{$70-80$} \\
\hline$t$ & 71.84 & 66.51 & 43.62 & 45.43 & 29.29 & \\
\hline$b$ & 18.66 & 19.08 & 12.95 & 20.10 & 13.53 & \\
\hline st & 20.41 & 15.87 & 8.73 & 6.64 & 2.58 & \\
\hline$s b$ & 3.31 & 2.64 & 2.05 & 3.04 & 1.76 & \\
\hline$\chi^{2}$ & 2.33 & 0.18 & 0.94 & 3.48 & 0.18 & \\
\hline$d$ & 108.00 & 90.00 & 68.00 & 59.00 & 45.00 & \multirow{6}{*}{$80-90$} \\
\hline$t$ & 80.27 & 65.24 & 40.57 & 40.68 & 27.14 & \\
\hline$b$ & 18.25 & 19.25 & 15.31 & 20.25 & 13.42 & \\
\hline st & 23.20 & 16.15 & 7.96 & 6.14 & 2.34 & \\
\hline$s b$ & 2.58 & 6.27 & 2.10 & 5.37 & 3.36 & \\
\hline$\chi^{2}$ & 0.67 & 0.27 & 1.88 & 0.05 & 0.39 & \\
\hline$d$ & 124.00 & 66.00 & 57.00 & 70.00 & 43.00 & \multirow{6}{*}{$90-100$} \\
\hline$t$ & 89.71 & 58.05 & 36.40 & 41.43 & 24.34 & \\
\hline$b$ & 22.62 & 16.43 & 13.26 & 19.03 & 11.20 & \\
\hline st & 27.32 & 14.95 & 6.96 & 6.37 & 2.20 & \\
\hline$s b$ & 4.69 & 2.44 & 1.84 & 4.70 & 1.72 & \\
\hline$\chi^{2}$ & 0.87 & 0.86 & 0.82 & 1.12 & 1.19 & \\
\hline$d$ & 238.00 & 102.00 & 81.00 & 102.00 & 65.00 & \multirow{6}{*}{$100-120$} \\
\hline$t$ & 160.38 & 102.87 & 63.44 & 67.97 & 46.53 & \\
\hline$b$ & 42.42 & 27.40 & 21.50 & 22.22 & 24.31 & \\
\hline st & 50.24 & 26.71 & 13.13 & 10.37 & 4.42 & \\
\hline$s b$ & 11.64 & 9.63 & 5.85 & 3.14 & 8.20 & \\
\hline$\chi^{2}$ & 4.13 & 5.78 & 0.16 & 1.21 & 0.44 & \\
\hline$d$ & 275.00 & 132.00 & 90.00 & 118.00 & 76.00 & \multirow{6}{*}{$120-150$} \\
\hline$t$ & 202.73 & 106.91 & 69.34 & 76.44 & 45.79 & \\
\hline$b$ & 49.23 & 27.36 & 20.34 & 29.73 & 21.93 & \\
\hline st & 67.44 & 28.26 & 14.96 & 12.05 & 4.42 & \\
\hline$s b$ & 16.53 & 5.68 & 3.87 & 7.39 & 3.83 & \\
\hline$\chi^{2}$ & 1.48 & 0.03 & 0.00 & 1.02 & 0.81 & \\
\hline$d$ & 171.00 & 100.00 & 47.00 & 57.00 & 47.00 & \multirow{6}{*}{$150-180$} \\
\hline$t$ & 146.20 & 66.22 & 41.56 & 48.00 & 30.25 & \\
\hline$b$ & 28.00 & 14.98 & 10.94 & 16.38 & 13.20 & \\
\hline st & 50.51 & 17.80 & 8.92 & 8.05 & 2.95 & \\
\hline$s b$ & 6.79 & 3.13 & 1.74 & 4.54 & 2.84 & \\
\hline$\chi^{2}$ & 0.05 & 2.92 & 0.52 & 0.78 & 0.24 & \\
\hline$d$ & 116.00 & 59.00 & 34.00 & 51.00 & 30.00 & \multirow{6}{*}{$180-220$} \\
\hline$t$ & 97.90 & 48.54 & 31.36 & 29.00 & 22.00 & \\
\hline$b$ & 21.72 & 8.71 & 11.14 & 12.15 & 12.50 & \\
\hline st & 35.09 & 13.93 & 7.11 & 4.67 & 2.21 & \\
\hline$s b$ & 3.81 & 0.83 & 3.44 & 2.11 & 3.91 & \\
\hline$\chi^{2}$ & 0.08 & 0.04 & 1.62 & 1.68 & 0.56 & \\
\hline$d$ & 87.00 & 34.00 & 18.00 & 19.00 & 22.00 & \multirow{6}{*}{$220-290$} \\
\hline$t$ & 59.25 & 31.56 & 15.88 & 23.05 & 18.45 & \\
\hline$b$ & 14.82 & 7.68 & 4.56 & 7.26 & 6.01 & \\
\hline st & 22.06 & 9.24 & 3.79 & 4.16 & 2.19 & \\
\hline$s b$ & 4.22 & 2.04 & 0.49 & 1.42 & 0.67 & \\
\hline$\chi^{2}$ & 1.48 & 0.61 & 0.27 & 5.20 & 0.24 & \\
\hline
\end{tabular}


Table A2: $\left(x, E_{\bar{\nu}}\right)$ distribution (20 data points).

\begin{tabular}{|l|r|r|r|r|r|c|}
\hline$x:$ & $0-0.10$ & $0.10-0.15$ & $0.15-0.20$ & $0.20-0.25$ & $0.25-0.50$ & $E_{\bar{\nu}}$ \\
\hline$d$ & 20.00 & 47.00 & 29.00 & 20.00 & 31.00 & \\
$t$ & 14.94 & 44.28 & 27.57 & 13.78 & 13.54 & \\
$b$ & 5.90 & 9.86 & 7.05 & 5.74 & 7.08 & \\
$s t$ & 3.41 & 7.45 & 3.41 & 1.09 & 0.60 & $35-60$ \\
$s b$ & 0.42 & 0.64 & 0.56 & 0.46 & 0.50 & \\
$\chi^{2}$ & 0.03 & 0.93 & 0.96 & 0.01 & 3.36 & \\
\hline$d$ & 64.00 & 57.00 & 27.00 & 20.00 & 19.00 & \\
$t$ & 61.27 & 50.42 & 32.03 & 17.13 & 15.44 & \\
$b$ & 14.26 & 9.38 & 4.29 & 3.29 & 5.34 & \\
$s t$ & 15.43 & 9.48 & 4.55 & 1.73 & 0.85 & $60-80$ \\
$s b$ & 1.74 & 0.75 & 0.47 & 0.22 & 0.46 & \\
$\chi^{2}$ & 1.64 & 0.11 & 2.71 & 0.01 & 0.15 & \\
\hline$d$ & 113.00 & 73.00 & 36.00 & 20.00 & 31.00 & \\
$t$ & 92.98 & 53.95 & 30.08 & 15.97 & 18.66 & \\
$b$ & 18.76 & 7.04 & 3.93 & 3.55 & 5.41 & \\
$s t$ & 26.59 & 10.77 & 4.31 & 1.60 & 1.22 & $80-110$ \\
$s b$ & 1.60 & 0.59 & 0.26 & 0.21 & 0.34 & \\
$\chi^{2}$ & 0.01 & 1.71 & 0.10 & 0.01 & 1.48 & \\
\hline$d$ & 145.00 & 47.00 & 20.00 & 27.00 & 25.00 & \\
$t$ & 102.09 & 47.64 & 26.88 & 15.69 & 16.31 & \\
$b$ & 35.11 & 5.08 & 3.53 & 2.67 & 5.04 & \\
$s t$ & 33.12 & 10.60 & 4.45 & 1.86 & 1.13 & $110-290$ \\
$s b$ & 4.00 & 0.34 & 0.23 & 0.18 & 0.55 & \\
$\chi^{2}$ & 0.33 & 0.56 & 4.39 & 2.57 & 0.50 & \\
& & & & & & \\
\hline
\end{tabular}

Table A3: $z_{l}$ distribution for neutrino (10 data points).

\begin{tabular}{|l|r|r|r|r|r|}
\hline$z_{l}:$ & $0.0-0.14$ & $0.14-0.18$ & $0.18-0.22$ & $0.22-0.26$ & $0.26-0.30$ \\
\hline$d$ & 551.00 & 412.00 & 432.00 & 433.00 & 413.00 \\
$t$ & 281.32 & 265.73 & 308.78 & 314.43 & 315.66 \\
$b$ & 291.77 & 131.64 & 113.66 & 101.12 & 70.82 \\
$s t$ & 61.78 & 60.29 & 72.08 & 72.44 & 73.14 \\
$s b$ & 97.40 & 32.15 & 23.55 & 20.28 & 10.34 \\
$\chi^{2}$ & 2.12 & 0.01 & 0.02 & 0.04 & 0.39 \\
\hline \hline$z_{l}:$ & $0.30-0.34$ & $0.34-0.38$ & $0.38-0.44$ & $0.44-0.54$ & $0.54-1.00$ \\
\hline$d$ & 366.00 & 330.00 & 370.00 & 411.00 & 393.00 \\
$t$ & 280.55 & 252.09 & 331.05 & 370.96 & 269.92 \\
$b$ & 53.55 & 46.41 & 50.98 & 61.09 & 74.71 \\
$s t$ & 65.28 & 60.69 & 81.05 & 90.84 & 64.51 \\
$s b$ & 6.21 & 6.04 & 5.47 & 6.46 & 6.72 \\
$\chi^{2}$ & 0.99 & 1.16 & 1.19 & 2.21 & 2.86 \\
\hline
\end{tabular}

Table A4: $z_{l}$ distribution for anti-neutrino ( 5 data points).

\begin{tabular}{|l|r|r|r|r|r|}
\hline$z_{l}:$ & $0.0-0.16$ & $0.16-0.22$ & $0.22-0.26$ & $0.26-0.30$ & $0.30-0.34$ \\
\hline$d$ & 215.00 & 182.00 & 164.00 & 163.00 & 147.00 \\
$t$ & 181.03 & 159.59 & 139.18 & 126.02 & 105.45 \\
$b$ & 51.83 & 27.32 & 19.00 & 20.36 & 44.24 \\
$s t$ & 36.90 & 31.37 & 27.83 & 26.27 & 21.47 \\
$s b$ & 5.41 & 2.32 & 1.52 & 1.54 & 3.80 \\
$\chi^{2}$ & 1.14 & 0.08 & 0.21 & 1.55 & 0.03 \\
\hline
\end{tabular}


Table A5: Correlation matrix of the fitted parameters.

\begin{tabular}{|c|c|c|c|c|c|}
\hline & $m_{c}$ & $\kappa$ & $\alpha$ & $B_{c}$ & $\epsilon$ \\
\hline$m_{c}$ & 1 & 0.423 & -0.291 & 0.229 & -0.276 \\
$\kappa$ & & 1 & -0.238 & -0.721 & -0.207 \\
$\alpha$ & & & 1 & 0.243 & -0.156 \\
$B_{c}$ & & & & 1 & 0.081 \\
$\epsilon_{P}$ & & & & & 1 \\
\hline
\end{tabular}

\section{References}

[1] A. Benvenuti et al., Phys. Rev. Lett. 34 (1975) 419.

[2] R. B. Barnett, Phys. Rev. Lett. 76 (1976) 1163.

[3] J. van Krogh et al., Phys. Rev. Lett. 36 (1976) 710.

[4] J. Blietschau et al., Phys. Rev. Lett. 60B (1976) 207.

[5] A. Asratyan et al., Phys. Lett. 71B (1977) 439.

[6] K. De Winter et al., Nucl. Inst. and Meth. A278 (1989) 670.

[7] M. A. G. Aivazis et al., Phys. Rev. D50 (1994) 3085; D50 (1994) 3102.

[8] D. Yu. Bardin, Preprint JINR-E2-86-260-mc (1986)

[9] Review of Particle properties, Phys. Rev. D54 (1996).

[10] V. N. Gribov and L. N. Lipatov, Sov. J. Nucl. Phys. 15 (1972) 438.

[11] G. Altarelli and G. Parisi, Nucl. Phys. B126 (1977) 298.

[12] S. A. Rabinowitz et al., CCFR Collaboration, Phys. Rev. Lett. 70 (1993) 134.

[13] H. L. Lai et al., Phys Rev. D51 (1995) 4763.

[14] C. Peterson et al., Phys. Rev. D27 (1983) 105.

[15] N. Ushida et al., Collaboration E531, Phys. Lett. B206 (1988) 375; B206 (1988) 375.

[16] H. Abramowicz et al., CDHS Collaboration, Z. Phys. C15 (1982) 19.

[17] T. Bolton, Preprint KSU-HEP-97-04 (1997), hep-ex/9708014.

[18] Review of Particles properties, Phys. Rev. D50 (1994).

[19] J. M. Conrad, M. H. Shaevitz, T. Bolton, Preprint KSU-HEP-97-04 (1997), hepex/9707015, to be published in Rev. Mod. Phys.

[20] D. Geiregat et al., Collaboration CHARM II, Phys. Lett. B245 (1990) 271.

[21] P. Vilain et al., Collaboration CHARM II, Phys. Lett. B313 (1993) 267.

[22] S. Adler, Phys. Rev. B135 (1964) 963.

[23] G. Ingelman, J. Rathsman, A. Edin, Preprint DESY 96-057, 1996, (http://www3.tsl.uu.se/thep/lepto/).

[24] GEANT, Detector Description and Simulation Tool, IT/ASD Group, CERN Program Library, Long Write-up Q123, 1995, (http://consult.cern.ch/writeups/geant).

[25] D. Geiregat et al., CHARM II Collaboration, Nucl. Inst. and Meth. A325 (1993) 92.

[26] W.M. Yeager et al., Phys. Rev. D16 (1977) 1294.

[27] T. H. Burnett et al., Phys. Lett. B77 (1978) 443.

[28] W. Wittek et al., Collaboration WA59, Z. Phys. C40 (1988) 231.

[29] M. Glück, E. Reya and A. Vogt, Z. Phys. C67 (1995) 433.

[30] K.S. McFarland, private communication.

[31] P. Collins et T. Spiller, J. Phys. G11 (1985) 1289.

[32] A.O. Bazarko et al., CCFR Collaboration, Z. Phys. C65 (1995) 189.

[33] S. Dasu et al., Phys. Rev. Lett. 61 (1988) 1061.

[34] H. Abramowicz et al., CDHS Collaboration, Phys. Rev. Lett. 57 (1986) 298;

A. Blondel et al., CDHS Collaboration, Z. Phys. C45 (1990) 361. 
[35] J. V. Allaby et al., CHARM Collaboration, Phys. Lett. B177 (1986) 446;

J. V. Allaby et al., CHARM Collaboration, Z. Phys. C36 (1987) 611.

[36] K. S. McFarland et al., CCFR/NuTeV collaboration, FNAL-Pub-97/001-E (1997).

[37] D. Bardin, private communication.

[38] D. Ward, the talk at 1997 International Europhysics Conference on High-energy Physics, 19-26 Aug, EPS-HEP '97 Jerusalem, Israel, hep-ph/9711515;

A. Martinez et al, Preprint CERN-EP/98-27, submitted to Rev. Mod. Phys. 